\title{
Pricing forward contracts in power markets by the certainty equivalence principle: Explaining the sign of the market risk premium is
}

\author{
Fred Espen Benth ${ }^{\mathrm{a}, *}$, Álvaro Cartea ${ }^{\mathrm{b}, 1}$, Rüdiger Kiesel \\ ${ }^{a}$ Centre of Mathematics for Applications, University of Oslo, Norway \\ ${ }^{\mathrm{b}}$ Commodities Finance Centre Birkbeck, University of London, UK \\ ${ }^{\mathrm{c}}$ Institute of Mathematical Finance, University of Ulm, Germany
}

\begin{abstract}
In this paper we provide a framework that explains how the market risk premium, defined as the difference between forward prices and spot forecasts, depends on the risk preferences of market players and the interaction between buyers and sellers. In commodities markets this premium is an important indicator of the behavior of buyers and sellers and their views on the market spanning between short-term and long-term horizons. We show that under certain assumptions it is possible to derive explicit solutions that link levels of risk aversion and market power with market prices of risk and the market risk premium. We apply our model to the German electricity market and show that the market risk premium exhibits a term structure which can be explained by the combination of two factors. Firstly, the levels of risk aversion of buyers and sellers, and secondly, how the market power of producers, relative to that of buyers, affects forward prices with different delivery periods.
\end{abstract}

JEL classification: G13; G11; D81; Q40

Keywords: Contango; Backwardation; Market price of risk; Electricity forwards; Market risk premium; Forward risk premium; Forward bias; Market power

\section{Introduction}

Commodities are a very different asset class from the more traditional classes of traded assets such as equities and bonds. Commodities normally encompass physical goods such as oil, gas, electricity, metals, agriculturals

\footnotetext{
This paper was reviewed and accepted while Prof. Giorgio Szego was the Managing Editor of The Journal of Banking and Finance and by the past Editorial Board. The authors are grateful to Kevin Metka, for excellent research assistance, and to two anonymous referees for their helpful comments.

Corresponding author. Tel.: +47 22855892; fax: +47 22854349.

E-mail addresses: fredb@math.uio.no (F.E. Benth), a.cartea@bbk. ac.uk (Á. Cartea), kiesel@mathematik.uni-ulm.de (R. Kiesel).

${ }^{1}$ Á. Cartea is thankful for the hospitality and generosity shown by the Finance Group at the Saïd Business School, Oxford, where part of this research was undertaken.
}

and live stock. The physical nature of commodities is perhaps one of their most defining characteristics specifically because it plays an important role in the behavior of their prices in both the spot and forward markets.

Let us contrast equity forwards with commodity forwards. For example, if interest rates and dividends are assumed to be deterministic, the pricing of equity forwards is a straightforward exercise. Simple no-arbitrage arguments are employed and the pricing is principally based on the ability to borrow money to purchase the underlying equity and hold it until delivery. As a result, the arbitrage-free forward price is the cost of borrowing net of dividends yielded by the equity. With commodities one can in principle apply a similar strategy to price forward contracts. However, the physical nature of commodities makes it very difficult for two reasons. First, the cost-of-carry (interest plus 'storage' costs) is not straightforward to calculate or measure. Second, it is 
necessary to account for the convenience yield (equivalent to collection of dividends on equities), but this is also exceptionally difficult to quantify or model.

The shape of commodities' forward curves for different delivery periods has always been of utmost importance to understand market players' (producers, consumers and speculators) 'attitudes' towards bearing risk in these markets. Forwards exhibit peculiar behavior depending on the time or delivery period. A situation where forward prices are above current spot prices is labeled contango and it is normally associated with circumstances where the immediate supply of the commodity is plentiful relative to demand. Similarly, the situation where forward prices are below spot prices is known as backwardation and it is generally associated with circumstances of low current supply levels and/or low inventory levels. One can determine whether contango or backwardation exists by simple observation of the forward markets. For example, in electricity and gas markets one normally observes that, for 'long' dated forward contracts, markets are in backwardation and for 'shorter' maturities the market is in contango (Cartea and Figueroa, 2005; Cartea and Williams, 2007).

Another quantity of importance that relates forward and expected spot prices is the market risk premium or forward bias $\pi(t, T)$. This is defined as the difference, calculated at time $t$, between the forward price $F(t, T)$, at time $t$ with delivery at $T$, and the expected spot price:

$\pi(t, T)=F(t, T)-\mathbb{E}^{P}\left[S(T) \mid \mathscr{F}_{t}\right]$.

Here $\mathbb{E}^{P}$ is the expectation operator, under the historical measure $P$, with information up until time $t$ and $S(T)$ is the spot price at time $T .^{2}$

To the best of our knowledge, recent literature on commodities has not addressed the connection between the market risk premium and market players' behavior and risk preferences. Moreover, it has not dealt with the question of why and how in some commodities markets we expect the market risk premium $\pi(t, T)$ to change signs in time $T$. The main contribution of this article is therefore to address these questions and propose a framework that allows us to establish explicit relationships between the market risk premium, the market price of risk and market players' risk preferences. By doing so, this allows us to explain the interesting connections between forward price formation and its deviations from spot forecasts based on the consumers' and producers' attitudes to risk.

To understand the importance of the market risk premium, it is important to point out that forward curves are not forecasts of the commodity spot price in the future. The clearing prices of forwards are the result of demand and supply, which in turn are determined by the individual characteristics of market players. Indeed the main motivation for players to engage in forward contracts is that of

\footnotetext{
${ }^{2}$ Note that it is incorrect to say that when $\pi(t, T)<0($ resp. $\pi(t, T)>0)$ the forward curve is in contango (resp. backwardation). Moreover, $S(t)$ is not generally a martingale under $P$.
}

risk diversification. Producers have made large investments with the aim of recouping them over a long period of time as well as making a return on them. As with any other investments, there is an incentive for producers to reduce variability in their profits by trading in instruments with payoffs that covary with their profits. Similarly, consumers (which might be intermediaries and/or use the commodity in their production process) also have an incentive to hedge their positions in the market by contracting forwards that help diversify their risks.

The relative appetite of producers and consumers for risk-diversification has a temporal dimension to it. Variations in this appetite for risk diversification will be evident in the different levels of market exposure chosen by producers and consumers and in the different levels chosen by members within each of these groups. For example a producer will generally be exposed to market uncertainty for a longer period of time, perhaps determined by the remaining life of its assets, whilst consumers will tend to make decisions based on a shorter time scale. In other words, the gains in terms of risk-diversification for consumers and producers will vary across time, therefore having a first order impact on forward clearing prices.

In this article we argue that it is precisely these differences in the desire to hedge positions and diversify risk that explain the market risk premium and its sign. Intuitively, the further out one looks into the market, the less incentivized consumers are to contract commodity forwards; however the producers' desire to hedge does not diminish as quickly. We associate situations where $\pi(t, T)>0$ (a positive market risk premium) with markets where the consumers' desire to cover their positions 'outweighs that of the producers. Conversely situations where $\pi(t, T)<0$ (a negative market risk premium) result when the producers' desire to hedge their positions outweighs that of the consumers.

In order to explain the market risk premium and the driving forces that give rise to it we organize the rest of the article as follows. Section 2 discusses the notion of a representative producer and a representative consumer. Based on their preferences we calculate an attainable set of forward prices where the two representative agents are willing to trade forward contracts. Section 3 discusses clearing market forward prices and the relative 'market power' agents have over these prices. Section 4 examines the market price of risk and market risk premium implied by our model under different assumptions. Section 5 applies our model to German electricity data and Section 6 concludes.

\section{Representative agents, price dynamics and forward price bounds}

In this section we describe producers' and consumers' preferences via the utility function of two representative agents. As an example we look at the wholesale electricity markets where we model the dynamics of the spot price as a stochastic process. Agents must decide how to manage their exposure to the spot and forward markets for every 
future date $T$. A key question for the producer is how much of his future production, which cannot be predicted with total certainty, will he wish to sell on the forward market or, when the time comes, sell it on the spot market. Similarly, the consumer must decide how much of her future needs, which cannot be predicted with full certainty either, will be acquired via the forward markets and how much on the spot. Clearly, as described above, both agents have the incentive to enter the forward market in the interest of risk diversification. We approach this financial decision and equilibrium price formation in two steps. First, we determine the forward price that makes the agents indifferent between the forward and spot market and, second, we discuss how the relative willingness of producers and consumers to hedge their exposures determines market clearing prices.

We assume that the risk preferences of the representative agents are expressed in terms of an exponential utility function parameterized by the risk aversion constant $\gamma>0$;

$U(x)=1-\exp (-\gamma x)$.

We let $\gamma:=\gamma_{\mathrm{p}}$ for the producer and $\gamma:=\gamma_{\mathrm{c}}$ for the consumer. The two agents can choose whether to act in the spot or the forward market. The forward market consists of contracts delivering the spot (physically, or in money terms) over a given delivery period. Typical examples can be the electricity or gas markets. In the latter the forward contracts have a monthly delivery period, while in the electricity market, which will be the particular case discussed in the remaining of this article, the contracts may have different periods of settlement, ranging from daily, through weekly and up to even yearly.

We want to derive bounds for forward prices through the principle of certainty equivalence between the two markets. In particular, we will obtain an upper bound, given by the maximum price the consumer is willing to pay before switching to the spot market, and a lower bound given by the producer's lowest forward price he is willing to trade at before switching to the spot market. These two bounds restrict forward prices to a set of feasible forward equilibrium prices and we postpone until Section 3 the discussion of how market clearing forward prices are singled out from this feasible set.

\subsection{Producers and consumers forward price bounds}

Let $(\Omega, \mathscr{F}, P)$ be a probability space equipped with a filtration $\mathscr{F}_{t}$. Following Lucía and Schwartz (2002) and Benth et al. (2007) we assume that the electricity spot price follows a mean-reverting multi-factor additive process

$S_{t}=\Lambda(t)+\sum_{i=1}^{m} X_{i}(t)+\sum_{j=1}^{n} Y_{j}(t)$

where $\Lambda(t)$ is the deterministic seasonal spot price level, while $X_{i}(t)$ and $Y_{j}(t)$ are the solutions to the stochastic differential equations $\mathrm{d} X_{i}(t)=-\alpha_{i} X_{i}(t) \mathrm{d} t+\sigma_{i}(t) \mathrm{d} B_{i}(t)$,

and

$\mathrm{d} Y_{j}(t)=-\beta_{j} Y_{j}(t) \mathrm{d} t+\mathrm{d} L_{j}(t)$.

Here, $B_{i}(t), i=1, \ldots, m$, are standard independent Brownian motions and $L_{j}(t), j=1, \ldots, n$ are independent Lévy processes. ${ }^{3}$ Let $\sigma_{i}(t)$ be (possibly seasonal) deterministic volatility functions. The processes $Y_{j}(t)$ are zero-mean reverting processes responsible for the spikes or large deviations which revert at a fast rate $\beta_{j}>0$, while $X_{i}(t)$ are zeromean reverting processes that account for the normal variations in the spot price evolution with mean-reversion $\alpha_{i}>0$.

We suppose that the Lévy processes are exponentially integrable in the sense that there exists a constant $\kappa>0$ such that

$\int_{|z| \geqslant 1} \mathrm{e}^{\tilde{\kappa} z} \ell_{j}(\mathrm{~d} z)<\infty$,

for all $\widetilde{\kappa} \leqslant \kappa$ and $j=1, \ldots, n$. This implies that the spot price process $S(t)$ has exponential moments up to order $\kappa$, and that the log-moment generating functions defined by

$\phi_{j}(x)=\ln \mathbb{E}\left[\mathrm{e}^{x L_{j}(1)}\right], \quad j=1, \ldots, n$,

exist for $|x| \leqslant \kappa$, where $\ell_{j}$ is the Lévy measure of the process $L_{j}(t)$. In the sequel we shall assume that $\kappa$ is sufficiently large to make the necessary exponential moments of $L_{j}(t)$ finite.

Assume that the producer will deliver the spot over the time interval $\left[T_{1}, T_{2}\right]$. He has the choice to deliver the production in the spot market, where he faces uncertainty in the prices over the delivery period, or to sell a forward contract with delivery over the same period. The producer takes this decision at time $t \leqslant T_{1}$.

We determine the forward price that makes the producer indifferent between the two alternatives, denoted by $F_{\mathrm{pr}}\left(t, T_{1}, T_{2}\right)$, from the equation

$$
\begin{aligned}
1 & -\mathbb{E}^{P}\left[\exp \left(-\gamma_{\mathrm{p}} \int_{T_{1}}^{T_{2}} S(u) \mathrm{d} u\right) \mid \mathscr{F}_{t}\right] \\
& =1-\mathbb{E}^{P}\left[\exp \left(-\gamma_{\mathrm{p}}\left(T_{2}-T_{1}\right) F_{\mathrm{pr}}\left(t, T_{1}, T_{2}\right)\right) \mid \mathscr{F}_{t}\right],
\end{aligned}
$$

or equivalently,

$$
\begin{aligned}
F_{\mathrm{pr}}\left(t, T_{1}, T_{2}\right)= & -\frac{1}{\gamma_{\mathrm{p}}} \frac{1}{T_{2}-T_{1}} \\
& \times \ln \mathbb{E}^{P}\left[\exp \left(-\gamma_{\mathrm{p}} \int_{T_{1}}^{T_{2}} S(u) \mathrm{d} u\right) \mid \mathscr{F}_{t}\right],
\end{aligned}
$$

where for simplicity we have assumed that the risk-free interest rate is zero. Note that $\int_{T_{1}}^{T_{2}} S(u) \mathrm{d} u$ is what the pro-

\footnotetext{
${ }^{3}$ In commodities markets one can expect to observe seasonal jumps. In this case we may use inhomogeneous Lévy or Sato processes which are processes with independent increments (Cont and Tankov, 2004; Sato, 1999). Only minor technical changes in what follows are required.
} 
ducer collects from selling the commodity on the spot market over the delivery period $\left[T_{1}, T_{2}\right]$, while he receives $\left(T_{2}-T_{1}\right) F_{\mathrm{pr}}\left(t, T_{1}, T_{2}\right)$ from selling it on the forward market.

In the Proposition below we employ the spot dynamics (2.1) to explicitly calculate the indifference forward price. For ease of presentation we introduce the notation for the following functions. For $i=1, \ldots, m$ and $j=1, \ldots, n$, $\bar{\alpha}_{i}\left(s, T_{1}, T_{2}\right)=\left\{\begin{array}{l}\frac{1}{\alpha_{i}}\left(\mathrm{e}^{-\alpha_{i}\left(T_{1}-s\right)}-\mathrm{e}^{-\alpha_{i}\left(T_{2}-s\right)}\right), \quad s \leqslant T_{1}, \\ \frac{1}{\alpha_{i}}\left(1-\mathrm{e}^{-\alpha_{i}\left(T_{2}-s\right)}\right), \quad s \geqslant T_{1} .\end{array}\right.$

and

$\bar{\beta}_{j}\left(s, T_{1}, T_{2}\right)=\left\{\begin{array}{l}\frac{1}{\beta_{j}}\left(\mathrm{e}^{-\beta_{j}\left(T_{1}-s\right)}-\mathrm{e}^{-\beta_{j}\left(T_{2}-s\right)}\right), \quad s \leqslant T_{1}, \\ \frac{1}{\beta_{j}}\left(1-\mathrm{e}^{-\beta_{j}\left(T_{2}-s\right)}\right), \quad s \geqslant T_{1} .\end{array}\right.$

Proposition 2.1. The price for which the producer is indifferent between the forward and spot market is given by

$$
\begin{aligned}
F_{\mathrm{pr}}( & \left.t, T_{1}, T_{2}\right) \\
= & \frac{1}{T_{2}-T_{1}} \int_{T_{1}}^{T_{2}} \Lambda(u) \mathrm{d} u+\sum_{i=1}^{m} \frac{\bar{\alpha}_{i}\left(t, T_{1}, T_{2}\right)}{T_{2}-T_{1}} X_{i}(t) \\
& +\sum_{j=1}^{n} \frac{\bar{\beta}_{j}\left(t, T_{1}, T_{2}\right)}{T_{2}-T_{1}} Y_{j}(t)-\frac{\gamma_{\mathrm{p}}}{2\left(T_{2}-T_{1}\right)} \\
& \times \int_{t}^{T_{2}} \sum_{i=1}^{m} \sigma_{i}^{2}(s) \bar{\alpha}_{i}^{2}\left(s, T_{1}, T_{2}\right) \mathrm{d} s-\frac{1}{\gamma_{\mathrm{p}}} \frac{1}{T_{2}-T_{1}} \\
& \times \int_{t}^{T_{2}} \sum_{j=1}^{n} \phi_{j}\left(-\gamma_{\mathrm{p}} \bar{\beta}_{j}\left(s, T_{1}, T_{2}\right)\right) \mathrm{d} s,
\end{aligned}
$$

where $\bar{\alpha}_{i}$ and $\bar{\beta}_{j}$ are given by (2.7) and (2.8), respectively.

Proof. Suppose for simplicity that $m=n=1$. We calculate the conditional expectation in (2.6). First observe that

$\int_{T_{1}}^{T_{2}} S(u) \mathrm{d} u=\int_{T_{1}}^{T_{2}} \Lambda(u) \mathrm{d} u+\int_{T_{1}}^{T_{2}} X(u) \mathrm{d} u+\int_{T_{1}}^{T_{2}} Y(u) \mathrm{d} u$.

Inserting the explicit dynamics of $X(u)$ and appealing to the stochastic Fubini Theorem (see e.g. Protter, 1992), we find

$$
\begin{aligned}
\int_{T_{1}}^{T_{2}} X(u) \mathrm{d} u & =\int_{T_{1}}^{T_{2}}\left\{X(t) \mathrm{e}^{-\alpha(u-t)}+\int_{t}^{u} \sigma(s) \mathrm{e}^{-\alpha(u-s)} \mathrm{d} B_{s}\right\} \mathrm{d} u \\
& =X(t) \bar{\alpha}\left(t, T_{1}, T_{2}\right)+\int_{T_{1}}^{T_{2}} \int_{t}^{u} \sigma(s) \mathrm{e}^{-\alpha(u-s)} \mathrm{d} B_{s} \mathrm{~d} u \\
& =X(t) \bar{\alpha}\left(t, T_{1}, T_{2}\right)+\int_{t}^{T_{2}} \sigma(s) \bar{\alpha}\left(s, T_{1}, T_{2}\right) \mathrm{d} B_{s} .
\end{aligned}
$$

A similar calculation for $\int_{T_{1}}^{T_{2}} Y(u) \mathrm{d} u$ yields,

$\int_{T_{1}}^{T_{2}} Y(u) \mathrm{d} u=Y(t) \bar{\beta}\left(t, T_{1}, T_{2}\right)+\int_{t}^{T_{2}} \bar{\beta}\left(s, T_{1}, T_{2}\right) \mathrm{d} L(s)$.

Thus, since $X(t)$ and $Y(t)$ are measurable with respect to $\mathscr{F}_{t}$ and using the independent increment properties of the Brownian motion and the Lévy process, we get,

$$
\begin{aligned}
\mathbb{E}\left[\exp \left(-\gamma_{\mathrm{pr}} \int_{T_{1}}^{T_{2}} S(u) \mathrm{d} u\right) \mid \mathscr{F}_{t}\right] \\
=\exp \left(-\gamma_{\mathrm{pr}}\left(\int_{T_{1}}^{T_{2}} \Lambda(u) \mathrm{d} u+X(t) \bar{\alpha}\left(t, T_{1}, T_{2}\right)\right.\right. \\
\left.\left.\quad+Y(t) \bar{\beta}\left(t, T_{1}, T_{2}\right)\right)\right) \\
\quad \times \mathbb{E}\left[\exp \left(-\gamma_{\mathrm{pr}} \int_{t}^{T_{2}} \sigma(s) \bar{\alpha}\left(s, T_{1}, T_{2}\right) \mathrm{d} B_{s}\right)\right] \\
\quad \times \mathbb{E}\left[\exp \left(-\gamma_{\mathrm{pr}} \int_{t}^{T_{2}} \bar{\beta}\left(s, T_{1}, T_{2}\right) \mathrm{d} L(s)\right)\right], \\
=\exp \left(-\gamma_{\mathrm{pr}}\left(\int_{T_{1}}^{T_{2}} \Lambda(u) \mathrm{d} u+X(t) \bar{\alpha}\left(t, T_{1}, T_{2}\right)\right.\right. \\
\left.\left.\quad+Y(t) \bar{\beta}\left(t, T_{1}, T_{2}\right)\right)\right) \\
\quad \times \exp \left(\frac{1}{2} \gamma_{\mathrm{pr}}^{2} \int_{t}^{T_{2}} \sigma^{2}(s) \bar{\alpha}^{2}\left(s, T_{1}, T_{2}\right) \mathrm{d} s\right) \\
\quad \times \exp \left(\int_{t}^{T_{2}} \phi\left(-\gamma_{\mathrm{pr}} \bar{\beta}\left(s, T_{1}, T_{2}\right)\right) \mathrm{d} s\right) .
\end{aligned}
$$

Thus, the Proposition is proved after taking logarithms and dividing by the risk aversion and length of the delivery period.

Before proceeding we can interpret how jumps in the model affect the indifference price calculated in Proposition 2.1 for the producer. For simplicity, if we assume that for the jump processes $L_{j}(t), j=1, \cdots, n$, each process can only jump either up or down it is straightforward to see how $F_{\mathrm{pr}}\left(t, T_{1}, T_{2}\right)$ is affected by each jump process. Suppose $L_{j}(t)$ is a process of only positive jumps. Then, the logmoment generating function $\phi_{j}(x)$ of $L_{j}(t)$ is an increasing function with $\phi_{j}(0)=0$. Thus, when $x<0, \phi_{j}(x)<0$, and since $\bar{\beta}_{j}$ is positive, we have that the argument of $\phi_{j}(\cdot)$ in the indifference price of the producer is negative, and thus the jump process $L_{j}(t)$ causes an increase in the indifference forward price. On the other hand, if $L_{j}(t)$ only exhibits negative jumps, we see that the indifference price is pushed downwards. This is intuitively clear, because the producer is willing to accept lower forward prices when there is a risk of price drops in the spot market, whereas positive price spikes work to the advantage of the producer, and he will be more reluctant to enter forward contracts that miss opportunities where he might be better-off selling in the spot market.

The consumer will derive the indifference price from the incurred expenses in the spot or forward market, which entails

$$
\begin{aligned}
1 & -\mathbb{E}^{P}\left[\exp \left(-\gamma_{\mathrm{c}}\left(-\int_{T_{1}}^{T_{2}} S(u) \mathrm{d} u\right)\right) \mid \mathscr{F}_{t}\right] \\
& =1-\mathbb{E}^{P}\left[\exp \left(-\gamma_{\mathrm{c}}\left(-\left(T_{2}-T_{1}\right) F_{\mathrm{c}}\left(t, T_{1}, T_{2}\right)\right)\right) \mid \mathscr{F}_{t}\right],
\end{aligned}
$$


or,

$$
\begin{aligned}
F_{\mathrm{c}}\left(t, T_{1}, T_{2}\right)= & \frac{1}{\gamma_{\mathrm{c}}} \frac{1}{T_{2}-T_{1}} \\
& \times \ln \mathbb{E}^{P}\left[\exp \left(\gamma_{\mathrm{c}} \int_{T_{1}}^{T_{2}} S(u) \mathrm{d} u\right) \mid \mathscr{F}_{t}\right] .
\end{aligned}
$$

We calculate the following price for the consumer.

Proposition 2.2. The price that makes the consumer indifferent between the forward and the spot market is given by

$$
\begin{aligned}
F_{\mathrm{c}}\left(t, T_{1}, T_{2}\right)= & \frac{1}{T_{2}-T_{1}} \int_{T_{1}}^{T_{2}} \Lambda(u) \mathrm{d} u+\sum_{i=1}^{m} \frac{\bar{\alpha}_{i}\left(t, T_{1}, T_{2}\right)}{T_{2}-T_{1}} X_{i}(t) \\
& +\sum_{j=1}^{n} \frac{\bar{\beta}_{j}\left(t, T_{1}, T_{2}\right)}{T_{2}-T_{1}} Y_{j}(t)+\frac{\gamma_{\mathrm{c}}}{2\left(T_{2}-T_{1}\right)} \\
& \times \int_{t}^{T_{2}} \sum_{i=1}^{m} \sigma_{i}^{2}(s) \bar{\alpha}_{i}^{2}\left(s, T_{1}, T_{2}\right) \mathrm{d} s+\frac{1}{\gamma_{\mathrm{c}}} \frac{1}{T_{2}-T_{1}} \\
& \times \int_{t}^{T_{2}} \sum_{j=1}^{n} \phi_{j}\left(\gamma_{\mathrm{c}} \bar{\beta}_{j}\left(s, T_{1}, T_{2}\right)\right) \mathrm{d} s .
\end{aligned}
$$

Proof. The proof is similar as in the producer's case.

Note that the producer prefers to sell his production in the forward market as long as the market forward price $F\left(t, T_{1}, T_{2}\right)$ is higher than $F_{\mathrm{pr}}\left(t, T_{1}, T_{2}\right)$. On the other hand, the consumer prefers the spot market if the market forward price is more expensive than his indifference price $F_{\mathrm{c}}\left(t, T_{1}, T_{2}\right)$. Thus, we have the bounds

$F_{\mathrm{pr}}\left(t, T_{1}, T_{2}\right) \leqslant F\left(t, T_{1}, T_{2}\right) \leqslant F_{\mathrm{c}}\left(t, T_{1}, T_{2}\right)$.

Letting the risk aversion of the producer go to zero, we end up with the expected earnings from selling in the spot market, also known as the forecasted forward price. We observe the same with the indifference price of the consumer when her risk aversion tends to zero.

Proposition 2.3. It holds

$$
\begin{aligned}
\lim _{\gamma_{p, c} \downarrow 0} & F_{\mathrm{pr}, \mathrm{c}}\left(t, T_{1}, T_{2}\right) \\
= & \mathbb{E}^{P}\left[\frac{1}{T_{2}-T_{1}} \int_{T_{1}}^{T_{2}} S(u) \mathrm{d} u \mid \mathscr{F}_{t}\right]=\frac{1}{T_{2}-T_{1}} \int_{T_{1}}^{T_{2}} \Lambda(u) \mathrm{d} u \\
& +\sum_{i=1}^{m} \frac{\bar{\alpha}_{i}\left(t, T_{1}, T_{2}\right)}{T_{2}-T_{1}} X_{i}(t)+\sum_{j=1}^{n} \frac{\bar{\beta}_{j}\left(t, T_{1}, T_{2}\right)}{T_{2}-T_{1}} Y_{j}(t) \\
& +\sum_{j=1}^{m} \frac{\phi_{j}^{\prime}(0)}{T_{2}-T_{1}} \int_{t}^{T_{2}} \bar{\beta}_{j}\left(s, T_{1}, T_{2}\right) \mathrm{d} s .
\end{aligned}
$$

Moreover, we find that

$$
\begin{aligned}
F_{\mathrm{pr}}\left(t, T_{1}, T_{2}\right) & \leqslant \mathbb{E}^{P}\left[\frac{1}{T_{2}-T_{1}} \int_{T_{1}}^{T_{2}} S(u) \mathrm{d} u \mid S(t)\right] \\
& \leqslant F_{\mathrm{c}}\left(t, T_{1}, T_{2}\right) .
\end{aligned}
$$

Proof. The first part is straightforward. The second part results from applying Jensen's inequality.

\section{Forward price and the market power}

Inequality (2.11) clearly indicates a range of prices where the producer and consumer are willing to attain a deal. Aggregate demand and supply will ultimately determine the clearing forward prices within this range. Previously we mentioned that the 'appetite' for risk diversification varies across consumers and producers, depending on their degree of risk-aversion. Moreover, it seems reasonable to assume that the desire to hedge exposure to market uncertainties will also vary with the horizon agents are looking at. In circumstances where there are not a large number of consumers hedging long-term positions, whilst at the same time producers are eager to hedge their exposure, we say that consumers have market power. Similarly, in situations (usually short-term horizons) where a large amount of consumers come to market to cover their positions, the balance of power tilts over to the producers.

We introduce the deterministic function $p\left(t, T_{1}, T_{2}\right) \in$ $[0,1]$ describing the market power of the representative producer which therefore depends on time $t$ and delivery period. If the producer has full market power, corresponding to $p\left(t, T_{1}, T_{2}\right)=1$, he can charge the maximum price possible in the forward market. This will be equal to the maximum price that the consumer can accept, namely $F_{\mathrm{c}}\left(t, T_{1}, T_{2}\right)$, since the consumer will leave the forward market for any higher price. On the other hand, if the consumer has full power, ie $p\left(t, T_{1}, T_{2}\right)=0$, she will drive the forward price as far down as possible which corresponds to $F_{\mathrm{pr}}\left(t, T_{1}, T_{2}\right)$. For any market power $0<p\left(t, T_{1}, T_{2}\right)<1$, the forward price $F^{p}\left(t, T_{1}, T_{2}\right)$ is defined to be

$$
\begin{aligned}
F^{p}\left(t, T_{1}, T_{2}\right)= & p\left(t, T_{1}, T_{2}\right) F_{\mathrm{c}}\left(t, T_{1}, T_{2}\right) \\
& +\left(1-p\left(t, T_{1}, T_{2}\right)\right) F_{\mathrm{pr}}\left(t, T_{1}, T_{2}\right) .
\end{aligned}
$$

In the most general setting, it would be possible to allow for a stochastic market power, being for instance dependent on the spot price dynamics. However, in this paper we shall constrain ourselves to the much simpler case of a deterministic market power. In some examples we consider it as a constant for simplicity, but in the empirical study of Section 5 we find evidence of a term structure for the market power.

The explicit dynamics for the forward price are easily stated as:

Proposition 3.1. The forward price dynamics are given by

$$
\begin{aligned}
F^{p}\left(t, T_{1}, T_{2}\right)= & \frac{1}{T_{2}-T_{1}} \int_{T_{1}}^{T_{2}} \Lambda(u) \mathrm{d} u+\sum_{i=1}^{m} \frac{\bar{\alpha}_{i}\left(t, T_{1}, T_{2}\right)}{T_{2}-T_{1}} X_{i}(t) \\
& +\sum_{j=1}^{n} \frac{\bar{\beta}_{j}\left(t, T_{1}, T_{2}\right)}{T_{2}-T_{1}} Y_{j}(t) \\
& +\frac{p\left(t, T_{1}, T_{2}\right)\left(\gamma_{\mathrm{pr}}+\gamma_{\mathrm{c}}\right)-\gamma_{\mathrm{pr}}}{2\left(T_{2}-T_{1}\right)} \\
& \times \int_{t}^{T_{2}} \sum_{i=1}^{m} \sigma_{i}^{2}(s) \bar{\alpha}_{i}^{2}\left(s, T_{1}, T_{2}\right) \mathrm{d} s
\end{aligned}
$$




$$
\begin{aligned}
& +\frac{p\left(t, T_{1}, T_{2}\right)}{\gamma_{\mathrm{c}}\left(T_{2}-T_{1}\right)} \int_{t}^{T_{2}} \sum_{j=1}^{n} \phi_{j}\left(\gamma_{\mathrm{c}} \bar{\beta}_{j}\left(s, T_{1}, T_{2}\right)\right) \mathrm{d} s \\
& -\frac{1-p\left(t, T_{1}, T_{2}\right)}{\gamma_{\mathrm{pr}}\left(T_{2}-T_{1}\right)} \\
& \times \int_{t}^{T_{2}} \sum_{j=1}^{n} \phi_{j}\left(-\gamma_{\mathrm{c}} \bar{\beta}_{j}\left(s, T_{1}, T_{2}\right)\right) \mathrm{d} s
\end{aligned}
$$

for $0 \leqslant t \leqslant T_{1}<T_{2}$.

Given the market power $p\left(t, T_{1}, T_{2}\right), F^{p}\left(t, T_{1}, T_{2}\right)$ is the price that the consumer and producer agree upon in the market. Since $p\left(t, T_{1}, T_{2}\right) \in[0,1]$, this forward price will be in between the producer's and the consumer's indifference price, so both are willing to accept such a price.

Let us discuss the correlation structure between forward contracts with different delivery periods. For simplicity we start with the case of no jumps $(m=0)$, and consider forward prices for two contracts with non-overlapping delivery periods $\left[T_{1}, T_{2}\right]$ and $\left[T_{3}, T_{4}\right]$. By the explicit form of $F^{p}\left(t, T_{1}, T_{2}\right)$ and the independence of the Brownian motions, we easily calculate

$$
\begin{aligned}
& \operatorname{Cov}\left(F^{p}\left(t, T_{1}, T_{2}\right), F^{p}\left(t, T_{3}, T_{4}\right)\right) \\
& =\sum_{i=1}^{m} \frac{\bar{\alpha}_{i}\left(t, T_{1}, T_{2}\right) \bar{\alpha}_{i}\left(t, T_{3}, T_{4}\right)}{\left(T_{2}-T_{1}\right)\left(T_{4}-T_{3}\right)} \int_{0}^{t} \sigma_{i}^{2}(s) \mathrm{e}^{-2 \alpha_{i}(t-s)} \mathrm{d} s .
\end{aligned}
$$

The correlation between two contracts can be estimated from market price data, and thus we can estimate the speeds of mean reversion $\alpha_{i}$ by calibrating the theoretical correlation to the empirical. Taking jumps into account will give rise to a structure where we can include the possibility of jump correlation between forward contracts. We see that it is the multi-factor spot model which implies the correlation structure for the forward contracts. This resembles the market models in fixed-income theory, the so-called LIBOR models, where one models each LIBOR rate separately, and include a correlation structure among the different rates. Further, it is also a known fact that forward contracts in energy markets are not perfectly correlated, but each contract has its intrinsic risk. Note that the market power function is not contributing to the correlation between two contracts.

\section{The market price of risk and market risk premium}

The standard way to price a forward contract is to find the conditional risk-neutral expected value of the future delivery from the contract. The risk-neutral probability is usually chosen to be related to what is called the market price of risk, which can be seen as a drift adjustment in the dynamics of an asset to reflect how investors are compensated for bearing risk when holding the asset. ${ }^{4}$ One of the peculiarities of commodities markets is that the market

\footnotetext{
${ }^{4}$ Note that the market price of risk is not what we have defined as the market risk premium.
}

price of risk may be either positive or negative depending on the time horizon considered. In Schwartz (1997) the calibration of one-factor models to futures prices of oil and copper delivered negative market prices of risk in both cases. Cartea and Figueroa (2005) model England and Wales wholesale electricity prices and estimate a negative market price of risk. Cartea and Williams (2007) model gas prices and forward contracts where a positive market price of risk for long-term contracts is observed and for short-term contracts the market price of risk, although positive on average, changes signs across time. In this section we want to relate the market power $p\left(t, T_{1}, T_{2}\right)$ to the market price of risk. By working with a parametrization of the market price of risk via a class of risk-neutral probabilities introduced by an Esscher transform, we shall see that there are explicit connections between the market power and both the market price of risk and the market risk premium. Further, to ensure an arbitrage-free forward market, we need to have certain conditions on the number of factors and contracts traded in the market. In commodities markets, especially electricity models, the connection between the physical and risk-neutral measure is usually performed by introducing a correction in the drift of the physical process to reflect how market participants are compensated for bearing risk, see for example (Schwartz, 1997; Schwartz and Smith, 2000; Lucía and Schwartz, 2002; Cartea and Figueroa, 2005; Cartea and Williams, 2007). We point out that this widely employed change of measure in the literature is the result of applying the Esscher transform to the physical measure which is what we propose to use in this article.

Suppose that we want to price a forward contract with delivery over the period $\left[T_{1}, T_{2}\right]$. The forward price is defined as

$F^{Q}\left(t, T_{1}, T_{2}\right)=\mathbb{E}^{Q}\left[\frac{1}{T_{2}-T_{1}} \int_{T_{1}}^{T_{2}} S(u) \mathrm{d} u \mid \mathscr{F}_{t}\right]$,

where we use $F^{Q}$ to indicate the dependency on the chosen risk-neutral probability $Q$.

We parameterize the market price of risk by introducing a probability measure $Q^{\theta}:==Q_{B} \times Q_{L}$, where $Q_{B}$ is a Girsanov transform of the Brownian motions $B_{i}(t), Q_{L}$ is an Esscher transform of the jump processes $L_{j}(t)$, and $\theta$ is an $\mathbb{R}^{n+m}$-valued function describing the market price of risk. We define the measure change as follows. For $t \leqslant T$, with $T \geqslant T_{2}$ being a finite time horizon encapsulating all the delivery periods in the market, let the probability $Q_{B}$ have the density process

$Z_{B}(t)=\exp \left(-\int_{0}^{t} \sum_{i=1}^{m} \frac{\theta_{B, i}(t)}{\sigma_{i}(s)} \mathrm{d} B_{i}(s)-\frac{1}{2} \int_{0}^{t} \sum_{i=1}^{m} \frac{\theta_{B, i}^{2}(s)}{\sigma_{i}^{2}(s)} \mathrm{d} s\right)$,

where we have supposed that the functions $\theta_{B, i} / \sigma_{i}$, $i=1, \ldots, m$, are square integrable over $[0, T]$. This measure change in the Wiener coordinates is given by the Girsanov transform, 
$\mathrm{d} W_{i}(t)=-\frac{\theta_{B, i}(t)}{\sigma_{i}(t)} \mathrm{d} t+\mathrm{d} B_{i}(t)$,

where $W_{i}(t)$ become Brownian motions on $[0, T], i=$ $1, \ldots, m$. The functions $\theta_{B, i}$ represent the compensation market players obtain for bearing the risk introduced by the non-extreme variations in the market, i.e. the diffusion component. We let it be time dependent to allow for variations across different seasons throughout the year. Later, we shall see that it could even be dependent on the delivery period, indicating that the market price of diffusion risk depends on the forward under consideration. This Girsanov change gives the dynamics (for $1 \leqslant i \leqslant m$ )

$\mathrm{d} X_{i}(t)=\left(\theta_{B, i}(t)-\alpha_{i} X_{i}(t)\right) \mathrm{d} t+\sigma_{i}(t) \mathrm{d} W_{i}(t)$,

and thus we have added a time-dependent level of meanreversion to the processes $X_{i}(t)$.

Further, define for bounded functions $\theta_{L, j}, j=1, \ldots, n$,

$Z_{L}(t)=\exp \left(\int_{0}^{t} \sum_{j=1}^{n} \theta_{L, j}(s) \mathrm{d} L_{j}(s)-\int_{0}^{t} \sum_{j=1}^{n} \phi_{j}\left(\theta_{L, j}(s)\right) \mathrm{d} s\right)$,

for $t \leqslant T_{2}$, and let the density process for the Radon-Nikodym derivative of the measure change in the jump component be

$\left.\frac{\mathrm{d} Q_{L}}{\mathrm{~d} P}\right|_{\mathscr{F}_{t}}=Z_{L}(t)$.

This is the so-called Esscher transform, and the time dependent functions $\theta_{L, j}(t)$ are the market prices of jump risk. We let $\theta:=\left(\theta_{B}, \theta_{L}\right)$, where $\theta_{B}:=\left(\theta_{B, i}\right)_{i=1}^{m}$ and $\theta_{L}:=\left(\theta_{L, j}\right)_{j=1}^{n}$. The density process of the probability $Q^{\theta}$ becomes $Z(t):=Z_{B}(t) Z_{L}(t)$. Further, we denote by $\mathbb{E}^{Q^{\theta}}$ the expectation with respect to the probability measure $Q^{\theta}$.

The forward price $F^{\theta}$ resulting from the market price of risk specification given by $Q^{\theta}$ is derived in the next Proposition.

Proposition 4.1. The forward price $F^{\theta}\left(t, T_{1}, T_{2}\right)$ is given by

$$
\begin{aligned}
F^{\theta}\left(t, T_{1}, T_{2}\right)= & \frac{1}{T_{2}-T_{1}} \int_{T_{1}}^{T_{2}} \Lambda(u) \mathrm{d} u \\
& +\sum_{i=1}^{m} \frac{\bar{\alpha}_{i}\left(t, T_{1}, T_{2}\right)}{T_{2}-T_{1}} X_{i}(t) \\
& +\sum_{j=1}^{n} \frac{\bar{\beta}_{j}\left(t, T_{1}, T_{2}\right)}{T_{2}-T_{1}} Y_{j}(t) \\
& +\int_{t}^{T_{2}} \sum_{i=1}^{m} \theta_{B, i}(s) \frac{\bar{\alpha}_{i}\left(s, T_{1}, T_{2}\right)}{T_{2}-T_{1}} \mathrm{~d} s \\
& +\int_{t}^{T_{2}} \sum_{j=1}^{n} \phi_{j}^{\prime}\left(\theta_{L, j}(s)\right) \frac{\bar{\beta}_{j}\left(s, T_{1}, T_{2}\right)}{T_{2}-T_{1}} \mathrm{~d} s .
\end{aligned}
$$

for $0 \leqslant t \leqslant T_{1}<T_{2}$.

Proof. For simplicity suppose $m=n=1$. In line with the calculations for the producer's and consumer's indifference prices, and from the explicit representation of $X(t)$ under $Q^{\theta}$ as

$$
\begin{aligned}
X(u)= & X(t) \mathrm{e}^{\alpha(u-t)}+\int_{t}^{u} \theta_{B}(s) \mathrm{e}^{-\alpha(u-s)} \mathrm{d} s \\
& +\int_{t}^{u} \sigma(u) \mathrm{e}^{-\alpha(u-s)} \mathrm{d} W(s),
\end{aligned}
$$

for $u \geqslant t$, we find

$$
\begin{aligned}
& \mathbb{E}^{Q^{\theta}}\left[\frac{1}{T_{2}-T_{1}} \int_{T_{1}}^{T_{2}} S(u) \mathrm{d} u \mid \mathscr{F}_{t}\right] \\
&=\frac{1}{T_{2}-T_{1}} \int_{T_{1}}^{T_{2}} \Lambda(u) \mathrm{d} u+X(t) \frac{\bar{\alpha}\left(t, T_{1}, T_{2}\right)}{T_{2}-T_{1}} \\
& \\
& \quad+Y(t) \frac{\bar{\beta}\left(t, T_{1}, T_{2}\right)}{T_{2}-T_{1}}+\frac{1}{T_{2}-T_{1}} \int_{T_{1}}^{T_{2}} \int_{t}^{u} \theta_{B}(s) \mathrm{e}^{-\alpha(u-s)} \mathrm{d} s \mathrm{~d} u \\
&+\frac{1}{T_{2}-T_{1}} \int_{T_{1}}^{T_{2}} \mathbb{E}^{Q_{L}}\left[\int_{t}^{u} \mathrm{e}^{-\beta(u-s)} \mathrm{d} L_{s} \mid \mathscr{F}_{t}\right] \mathrm{d} u .
\end{aligned}
$$

By Bayes' Theorem and the independent increment property of the Lévy process, we see that the expectation in the last integral is

$$
\begin{aligned}
& \mathbb{E}^{Q_{L}}\left[\int_{t}^{u} \mathrm{e}^{-\beta(u-s)} \mathrm{d} L(s) \mid \mathscr{F}_{t}\right] \\
& =\mathbb{E}^{P}\left[\int_{t}^{u} \mathrm{e}^{-\beta(u-s)} \mathrm{d} L(s) \frac{Z_{L}(u)}{Z_{L}(t)} \mid \mathscr{F}_{t}\right] \\
& =\mathbb{E}^{P}\left[\int_{t}^{u} \mathrm{e}^{-\beta(u-s)} \mathrm{d} L(s) \mathrm{e}^{\int_{t}^{u} \theta_{L}(s) \mathrm{d} L(s)-\int_{t}^{u} \phi\left(\theta_{L}(s)\right) \mathrm{d} s}\right] \\
& =\left.\frac{\mathrm{d}}{\mathrm{d} x} \mathbb{E}^{P}\left[\mathrm{e}^{\int_{t}^{u}\left(\mathrm{e}^{-\beta(u-s)}+\theta_{L}(s)\right) \mathrm{d} L(s)}\right]\right|_{x=0} \times \mathrm{e}^{-\int_{t}^{u} \phi\left(\theta_{L}(s)\right) \mathrm{d} s} \\
& =\left.\frac{\mathrm{d}}{\mathrm{d} x} \mathrm{e}_{t}^{u} \phi\left(x \mathrm{e}^{-\beta(u-s)}+\theta_{L}(s)\right) \mathrm{d} s\right|_{x=0} \times \mathrm{e}^{-\int_{t}^{u} \phi\left(\theta_{L}(s)\right) \mathrm{d} s} \\
& =\int_{t}^{u} \phi^{\prime}\left(\theta_{L}\right) \mathrm{e}^{-\beta(u-s)} \mathrm{d} s .
\end{aligned}
$$

After reorganizing the integrals the result follows.

To gain insight into how the market risk premium depends on diffusion and jump risk we look at the following two corollaries. The first one assumes that the market price of jump risk be zero. The second one assumes that the market price of diffusion risk is zero.

Corollary 4.2. Suppose that the market price of jump risk is zero, i.e. $\theta_{L, j}(s)=0$ for $j=1, \ldots, n$. Then

$$
\begin{aligned}
F^{\theta}\left(t, T_{1}, T_{2}\right)= & \mathbb{E}^{P}\left[\frac{1}{T_{2}-T_{1}} \int_{T_{1}}^{T_{2}} S(u) \mathrm{d} u \mid \mathscr{F}_{t}\right] \\
& +\int_{t}^{T_{2}} \sum_{i=1}^{m} \theta_{B, i}(s) \frac{\bar{\alpha}_{i}\left(s, T_{1}, T_{2}\right)}{T_{2}-T_{1}} \mathrm{~d} s .
\end{aligned}
$$

Proof. This is straightforward from the results above.

Thus, from this we see that when market players are not compensated for bearing jump risk, the market risk premium is positive as long as 
$\pi\left(t, T_{1}, T_{2}\right)=\int_{t}^{T_{2}} \sum_{i=1}^{m} \theta_{B, i}(s) \frac{\bar{\alpha}_{i}\left(s, T_{1}, T_{2}\right)}{T_{2}-T_{1}} \mathrm{~d} s$

is positive. As a particular case we can assume all $\theta_{B, i}(t)$ 's to be positive constants which yields a positive market price of risk since $\bar{\alpha}_{i}$ are positive functions for all $s \leqslant T_{2}$. However, in the more general setting, one can obtain a change in the sign of the market risk premium over time $t$ by appropriate specification of the functions $\theta_{B, i}(t)$. Furthermore, although changes in the sign of the market price of risk are also of particular interest, it is clear that a change in the sign of the market prices of risk $\theta_{B, i}(t)$ does not always imply a change in the sign of the market risk premium.

Now we turn our attention to the case with no diffusion market price of risk, i.e. $\theta_{B, i}(t)=0$ for all $i=1, \ldots, m$.

Corollary 4.3. Suppose that $\theta_{B, i}(t)=0$ for all $i=1, \ldots, m$. Then

$$
\begin{aligned}
F^{\theta}\left(t, T_{1}, T_{2}\right)= & \mathbb{E}^{P}\left[\frac{1}{T_{2}-T_{1}} \int_{T_{1}}^{T_{2}} S(u) \mathrm{d} u \mid \mathscr{F}_{t}\right] \\
& +\int_{t}^{T_{2}} \sum_{j=1}^{n}\left\{\phi_{j}^{\prime}\left(\theta_{L, j}(s)\right)-\phi_{j}^{\prime}(0)\right\} \frac{\bar{\beta}_{j}\left(s, T_{1}, T_{2}\right)}{T_{2}-T_{1}} \mathrm{~d} s .
\end{aligned}
$$

Proof. Follows from the results above.

Note that the market risk premium in this case is given by

$\pi\left(t, T_{1}, T_{2}\right)=\int_{t}^{T_{2}} \sum_{j=1}^{n}\left\{\phi_{j}^{\prime}\left(\theta_{L, j}(s)\right)-\phi_{j}^{\prime}(0)\right\} \frac{\bar{\beta}_{j}\left(s, T_{1}, T_{2}\right)}{T_{2}-T_{1}} \mathrm{~d} s$,

and even if we assume constant and positive $\theta_{L, j}>0$, the sign of $\pi\left(t, T_{1}, T_{2}\right)$ will depend on the monotonicity of $\phi_{j}^{\prime}$. In general, the sign of the market risk premium will result from a combination of $\theta_{L, j}(t)$ and the monotonicity properties of $\phi_{j}^{\prime}$.

Finally, when both the market price of jump and diffusion risk are taken into account, the market risk premium is given by

$$
\begin{aligned}
\pi\left(t, T_{1}, T_{2}\right)= & \int_{t}^{T_{2}} \sum_{i=1}^{m} \theta_{B, i}(s) \frac{\bar{\alpha}_{i}\left(s, T_{1}, T_{2}\right)}{T_{2}-T_{1}} \mathrm{~d} s \\
& +\int_{t}^{T_{2}} \sum_{j=1}^{n}\left\{\phi_{j}^{\prime}\left(\theta_{L, j}(s)\right)-\phi_{j}^{\prime}(0)\right\} \frac{\bar{\beta}_{j}\left(s, T_{1}, T_{2}\right)}{T_{2}-T_{1}} \mathrm{~d} s .
\end{aligned}
$$

Now we proceed to relate the market risk premium (4.2) to the market power $p\left(t, T_{1}, T_{2}\right)$. Comparing the expressions of $F^{\theta}$ given by (4.1) and $F^{p}$ calculated in Proposition 3.1, we have that the sum of the last two terms of $F^{p}$ must match the sum of the two last terms of $F^{\theta}$. Hence, we must find a solution $\theta=\left(\theta_{B}, \theta_{L}\right)$ to the equation

$$
\begin{aligned}
\int_{t}^{T_{2}} & \sum_{i=1}^{m} \theta_{B, i}(s) \bar{\alpha}_{i}\left(s, T_{1}, T_{2}\right) \mathrm{d} s \\
& +\int_{t}^{T_{2}} \sum_{j=1}^{n} \phi_{j}^{\prime}\left(\theta_{L, j}(s)\right) \bar{\beta}_{j}\left(s, T_{1}, T_{2}\right) \mathrm{d} s \\
= & \frac{1}{2}\left[p\left(t, T_{1}, T_{2}\right)\left(\gamma_{\mathrm{pr}}+\gamma_{\mathrm{c}}\right)-\gamma_{\mathrm{pr}}\right] \\
& \int_{t}^{T_{2}} \sum_{i=1}^{m} \sigma_{i}^{2}(s) \bar{\alpha}_{i}^{2}\left(s, T_{1}, T_{2}\right) \mathrm{d} s+\frac{p\left(t, T_{1}, T_{2}\right)}{\gamma_{\mathrm{c}}} \\
& \int_{t}^{T_{2}} \sum_{j=1}^{n} \phi_{j}\left(\gamma_{\mathrm{c}} \bar{\beta}_{j}\left(s, T_{1}, T_{2}\right)\right) \mathrm{d} s-\frac{1-p\left(t, T_{1}, T_{2}\right)}{\gamma_{\mathrm{pr}}} \\
& \int_{t}^{T_{2}} \sum_{j=1}^{n} \phi_{j}\left(-\gamma_{\mathrm{pr}} \bar{\beta}_{j}\left(s, T_{1}, T_{2}\right)\right) \mathrm{d} s .
\end{aligned}
$$

We have $n+m$ unknown functions $\theta_{B, i}$ and $\theta_{L, j}$, $i=1, \ldots, m, j=1, \ldots, n$. If the market consists of $k$ contracts with non-overlapping delivery periods, we may find a solution as long as $k \leqslant m+n$. We need at least one solution $\theta=\left(\theta_{B}, \theta_{L}\right)$ in order to have an arbitrage-free market of forward contracts. Suppose for instance that $n=0$, that is, there are no jumps in the market. In this case we have $k$ linear equations for the $m$ unknown functions where we find at least one solution as long as $k \leqslant m$ and the mean reversion coefficients $\alpha_{i}$ are different. If $m>0$ and $n>0$ we have both diffusion and jumps in the model, and we may simply choose $\theta_{L, j}$ freely, and then solve for the remaining unknowns $\theta_{B, j}$ given by Eq. (4.3) for each $k$ delivery periods. There is at least one solution when $k \leqslant m$ for this situation whenever the $\alpha_{i}$ 's are different. One way is to choose $\theta_{L, j}(t)=0$, which means that there is no price for jump risk incurred by the market, see for example (Merton, 1990). We remark in passing that if we chose a spot model with just two factors (for example $m=n=1$ ), and the market trades in more than two forward contracts, there may be no solution to the Eq. (4.3) for all the different contracts at once. Solving the equations in (4.3) for each contract separately leads to solutions which are dependent on the delivery period, and will not give one risk neutral measure for the market as a whole, but one measure for each contract separately; allowing for arbitrage opportunities.

In the rest of this section we look at two illustrative examples to gain further insights into the model before applying it to German data in Section 5. First we shall demonstrate that for a simple Poisson jump model and constant market power, the market risk premium changes sign across time. Second, we explore the case of fixed time delivery without the presence of jumps and look explicitly at how $\pi(t, T)$ depends on the parameters $p(t, T), \gamma_{\mathrm{pr}}$ and $\gamma_{\mathrm{c}}$.

\subsection{An example with constant market power and poisson jumps}

We consider a forward market consisting of 52 contracts with weekly delivery. The market power is supposed to be 
constant $p\left(t, T_{1}, T_{2}\right)=p \in[0,1]$. Assume that the spot model has $m=52$ diffusion components $X_{i}(t)$, and one $(n=1)$ jump component $Y(t)$. Suppose that the seasonal function is

$\Lambda(t)=150+20 \cos (2 \pi t / 365)$,

and the mean-reversion parameters for the diffusion components are $\alpha_{i}=0.067 / i$, with volatility $\sigma_{i}=0.3 / \sqrt{i}$, for $i=1, \ldots, 52$. We mimic here a sequence of mean-reverting processes with decreasing speeds of mean reversion and with decreasing volatility. Note that a speed of mean reversion equal to 0.067 means that a shock will be halved over 10 days. The jump process is driven by $L(t)=\eta N(t)$, where $N(t)$ is a Poisson process with intensity $\lambda$ and the jump size is constant, equal to $\eta$. The mean-reversion for the jump component is $\beta=0.5$, meaning that a jump will, on average, revert back in two days. Thus, we have a combination of slow mean reverting normal variations and fast mean reverting spikes in the spot market. The frequency of spikes is set to $\lambda=2 / 365$, i.e. two spikes, on average, per year. Time $t=0$ corresponds to January 1 , and we assume that the initial spot price is $S(0)=172$. In our empirical investigations, we let $X_{1}(0)=2$, and $X_{i}(0)=Y(0)=0$ for $i=2, \ldots, 52$ to achieve this. The risk aversion coefficients of the producer and consumer are set equal to $\gamma_{\mathrm{c}}=\gamma_{\mathrm{pr}}=0.5$. In the examples below we derive forward curves for weakly settled forward contracts over a year. We remark that this model is chosen for its simplicity and to illustrate the approach in this paper.

Consider first a positive jump of size $\eta=10$. In Fig. 1 we have plotted the indifference forward curves for the producer and consumer (' $x$ '), together with the forward curves with constant market power equal to $p=0.25,0.5$ and 0.75 (marked as dashed lines, in increasing order). Finally, we have included the forecasted spot price curve as ' + '. We clearly see that the forecasted price curve follows a shape similar to the seasonal function, while the indifference price curves have very different shapes in the short end. As expected, market clearing forward prices are increasing with increasing market power, since the producer will command higher prices with more power. Note also that for a low market power of 0.25 , we still observe that the forecasted price curve is below the forward curve in the shorter end, while in the medium to long end we see the opposite. This corresponds to a positive market risk premium in the shorter end, whereas it becomes negative in the medium and longer end. Both players have the same risk aversion, and the consumer wishes to avoid upward jumps in the price. Hence, even for a weak producer, the consumer is willing to accept a positive market risk premium in the short end. In the long end, the effect of jumps vanish as a consequence of mean reversion and the consumer will have more power driving the market risk premium below zero. To illustrate this particular example with $p=0.5$ we have plotted the difference of the forward curve with market power 0.25 and the forecasted curve in Fig. 2. For the contracts with delivery up to approximately week 20 , the market premium is positive. The premium decreases with time to delivery, and becomes negative in the medium and long end.

Turning our attention to the case of negative jumps, we observe the reverse picture. Suppose that jumps sizes are fixed at $\eta=-10$. Fig. 3 shows the corresponding forward and indifference curves together with the forecasted price. We observe first of all that all curves are shifted downwards, indicating that the producer is willing to accept lower forward prices to hedge the possibility of sudden drops in prices. In the short-term we observe, for all cases of market power, that the forecasted spot price is above forward prices, i.e. negative market risk premium. In the long-term, only when producer's market power is high, that is 0.75 , we have the situation where the forecasted curve is below the forward curve signaling that the consumer bears a positive risk premium. Moreover, Fig. 4

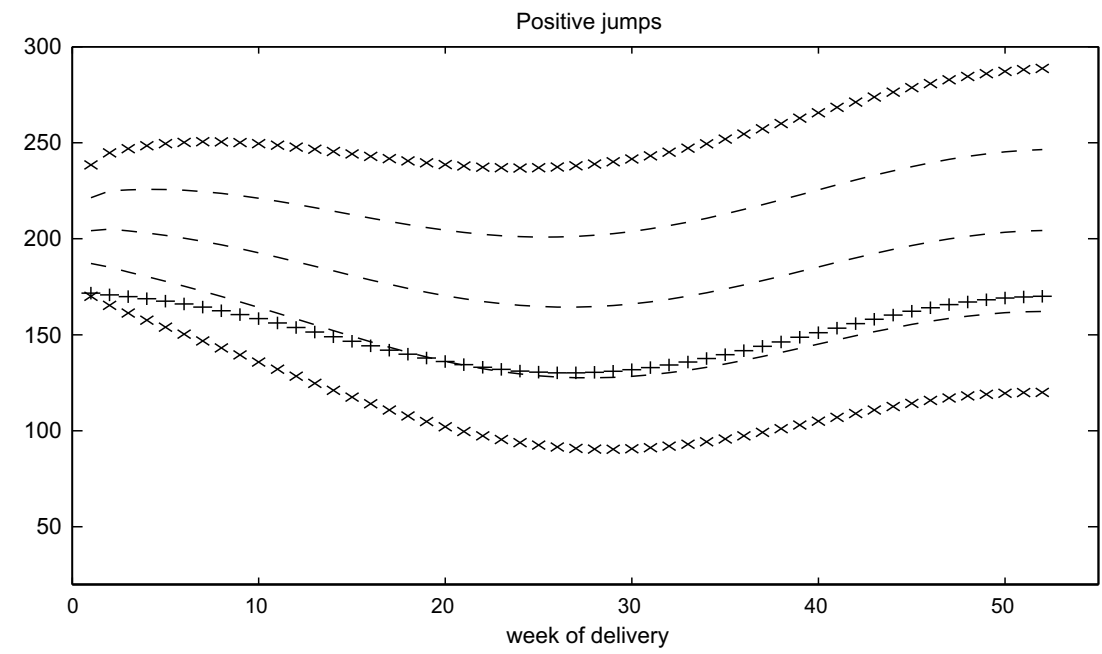

Fig. 1. The indifference price curves together with the forward curves for market powers equal to $p=0.25,0.5$ and $p=0.75$, in increasing order. The forecasted curve is depicted ' + '. The jumps are positive of size 10 . 


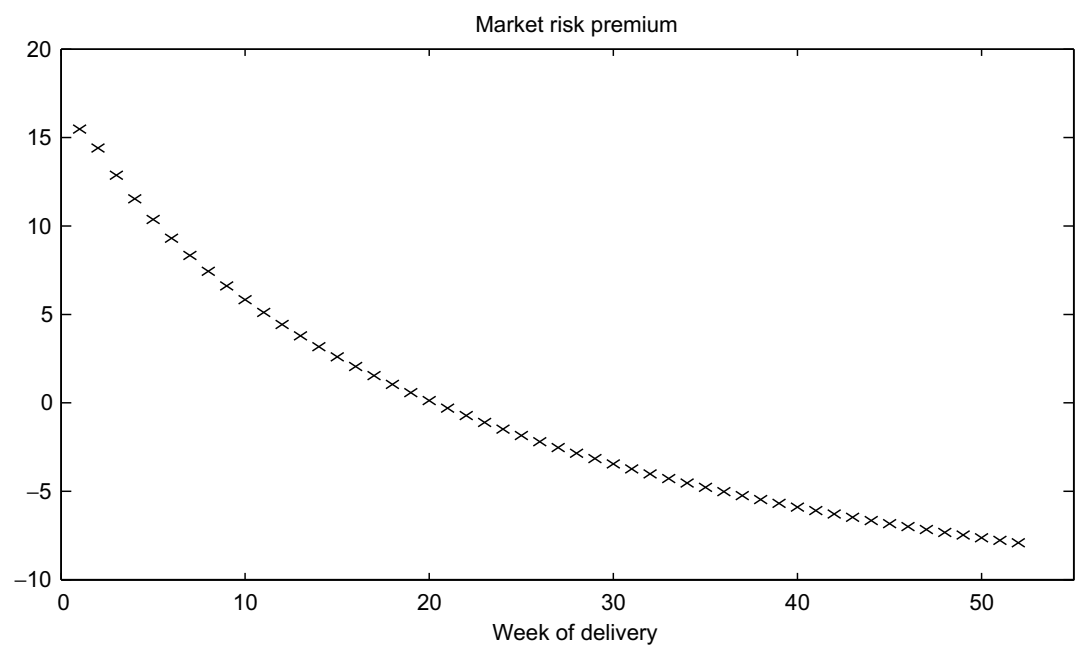

Fig. 2. The market risk premium given by the difference of the forward curve with market power 0.25 and the forecasted curve.

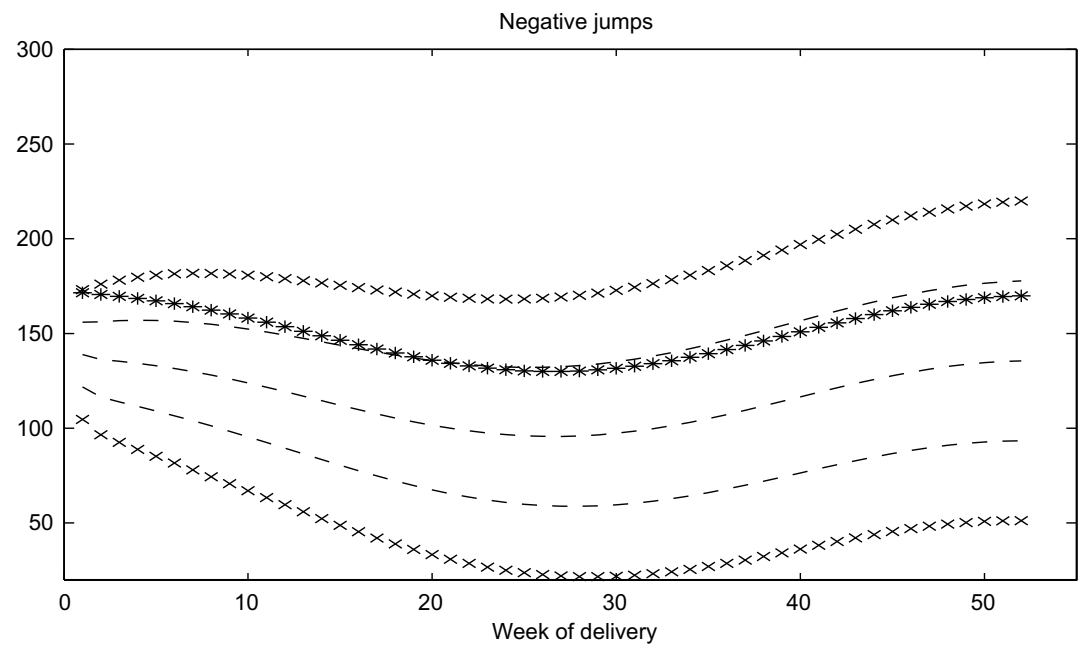

Fig. 3. The indifference price curves together with the forward curves for market powers equal to $p=0.25,0.5$ and $p=0.75$, in increasing order. The forecasted curve is depicted with ${ }^{* *}$. The jumps are negative of size 10.

shows the difference between the forward curve and the forecasted curve when the producer's market power is $p=0.75$.

We now proceed to analyze more closely the implications of jumps and normal variations of the model. We consider the case with $m=n=1$ and constant market power $p\left(t, T_{1}, T_{2}\right)=p$ for $p \in[0,1]$. Further, let $L(t)=N(t)$, a Poisson process with constant jump intensity $\lambda>0$. Note that this model has only two factors, and in general it will not give an arbitrage-free forward curve dynamics for a market which trades in contracts with many different delivery periods. However, this simplification provides us with some insight into how the sign of the market risk premium may change, and we include it with the assumption that we have one forward contract with delivery period $\left[T_{1}, T_{2}\right]$ traded in the market.
Consider Eq. (4.3). One way to solve this is to separate the Wiener and jump part, and solve the two resulting equations. We find the solution

$\theta_{B}\left(t, T_{1}, T_{2}\right)=\frac{1}{2}\left(p\left(\gamma_{\mathrm{pr}}+\gamma_{\mathrm{c}}\right)-\gamma_{\mathrm{pr}}\right) \sigma^{2}(t) \bar{\alpha}\left(t, T_{1}, T_{2}\right)$,

for $t \leqslant T_{2}$. Note that the sign of $\theta_{B}$ depends on the sign of $p\left(\gamma_{\mathrm{pr}}+\gamma_{\mathrm{c}}\right)-\gamma_{\mathrm{pr}}$, since $\sigma^{2}(t)$ and $\bar{\alpha}\left(t, T_{1}, T_{2}\right)$ are positive. We have a negative market price of risk $\theta_{B}$ whenever

$p<\frac{\gamma_{\mathrm{pr}}}{\gamma_{\mathrm{pr}}+\gamma_{\mathrm{c}}}$.

If for instance $\gamma_{\mathrm{pr}}=\gamma_{\mathrm{c}}$, the market price of risk $\theta_{B}$ becomes negative whenever $p<0.5$, which corresponds to the consumer being the strongest. If the producer is stronger, i.e. $p>0.5$, he is the superior power in forming prices and the 


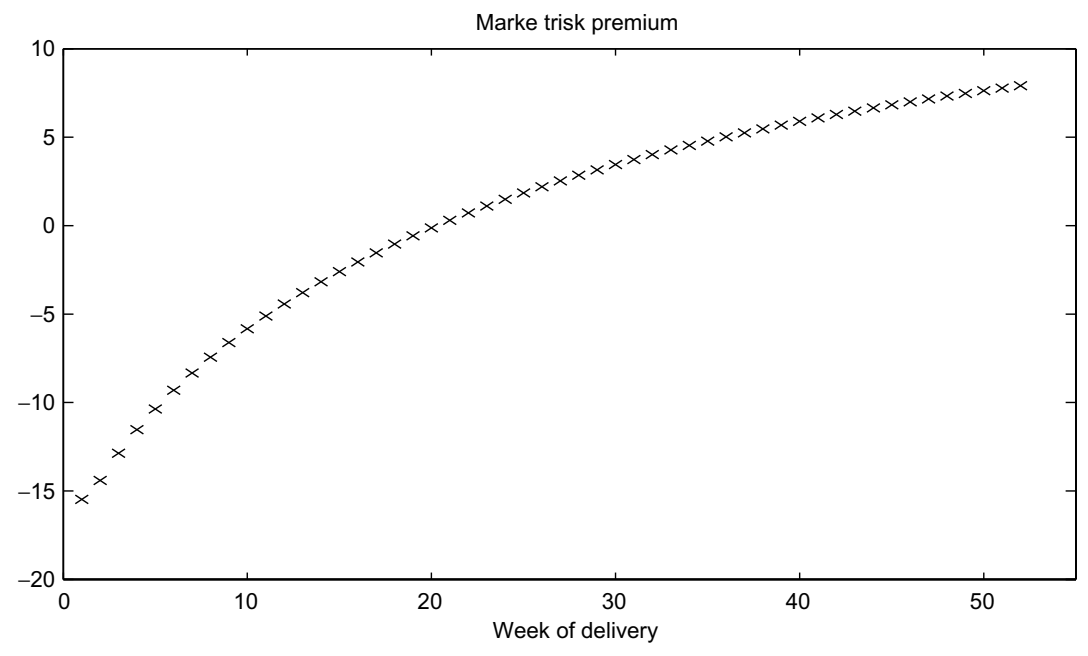

Fig. 4. The market risk premium given by the difference of the forward curve with market power 0.75 and the forecasted curve.

market price of risk becomes positive. If $\gamma_{\mathrm{pr}} \neq \gamma_{\mathrm{c}}$, the market power needs to be less than the relative risk aversion of the producer against the total risk aversion for $\theta_{B}$ to be negative.

Let us consider the market price of jump risk. Since $L(t)$ is assumed to be a Poisson process, the log-moment generating function is given by

$\phi(x)=\lambda\left(\mathrm{e}^{x}-1\right) \quad$ and $\quad \phi^{\prime}(x)=\lambda \mathrm{e}^{x}$.

Note that

$\phi^{\prime}\left(\theta_{L}(t)\right)-\phi^{\prime}(0)=\lambda\left(\mathrm{e}^{\theta_{L}(t)}-1\right)$

which is positive whenever $\theta_{L}(t)>0$, and negative if $\theta_{L}(t)<0$, as expected following the interpretation of Corollary 4.3. The equation for the jump risk derived from splitting (4.3) into two equations becomes (after differentiating with respect to $t$ )

$$
\begin{aligned}
\lambda \mathrm{e}^{\theta_{L}(t)} \bar{\beta}\left(t, T_{1}, T_{2}\right)= & \frac{p}{\gamma_{\mathrm{c}}} \lambda\left(\mathrm{e}^{\gamma_{\mathrm{c}} \bar{\beta}\left(t, T_{1}, T_{2}\right)}-1\right) \\
& -\frac{1-p}{\gamma_{\mathrm{pr}}} \lambda\left(\mathrm{e}^{-\gamma_{\mathrm{pr}} \bar{\beta}\left(t, T_{1}, T_{2}\right)}-1\right) .
\end{aligned}
$$

Or, equivalently,

$$
\begin{aligned}
\bar{\beta}\left(t, T_{1}, T_{2}\right) \mathrm{e}^{\theta_{L}\left(t, T_{1}, T_{2}\right)}= & \frac{p}{\gamma_{\mathrm{c}}}\left(\mathrm{e}^{\gamma_{\bar{\beta}} \bar{\beta}\left(t, T_{1}, T_{2}\right)}-1\right) \\
& +\frac{1-p}{\gamma_{\mathrm{pr}}}\left(1-\mathrm{e}^{-\gamma_{\mathrm{pr}} \bar{\beta}\left(t, T_{1}, T_{2}\right)}\right) .
\end{aligned}
$$

Note that the right-hand side of (4.6) is positive since $\bar{\beta}\left(t, T_{1}, T_{2}\right)>0$. Thus, the market price of jump risk is negative whenever

$\frac{p}{\gamma_{\mathrm{c}}}\left(\mathrm{e}^{\gamma_{\mathrm{c}} \bar{\beta}\left(t, T_{1}, T_{2}\right)}-1\right)+\frac{1-p}{\gamma_{\mathrm{pr}}}\left(1-\mathrm{e}^{-\gamma_{\mathrm{pr}} \bar{\beta}\left(t, T_{1}, T_{2}\right)}\right)<\bar{\beta}\left(t, T_{1}, T_{2}\right)$,

and positive otherwise. The following Lemma is helpful in understanding when the market price of jump risk is negative.
Lemma 4.4. The non-negative function $f:=\mathbb{R}_{+} \mapsto \mathbb{R}_{+}$ defined by

$f(z)=\frac{p}{\gamma_{\mathrm{c}}}\left(\mathrm{e}^{\gamma_{\mathrm{c}} z}-1\right)+\frac{1-p}{\gamma_{\mathrm{pr}}}\left(1-\mathrm{e}^{-\gamma_{\mathrm{pr}} z}\right)$,

satisfies $f(z) \geqslant z$ for all $z \geqslant 0$ when

$p>\frac{\gamma_{\mathrm{pr}}}{\gamma_{\mathrm{pr}}+\gamma_{\mathrm{c}}}$.

Moreover, if

$p<\frac{\gamma_{\mathrm{pr}}}{\gamma_{\mathrm{pr}}+\gamma_{\mathrm{c}}}$

then $f(z)<z$ for $z \leqslant z_{0}$, and $f(z) \geqslant z$ otherwise, where $z_{0}$ is defined by $f(z)=z$.

Proof. Observe that $f(0)=0$, and $f(z) \rightarrow \infty$ whenever $z \rightarrow \infty$. Moreover, $f$ is monotonically increasing since

$f^{\prime}(z)=p \mathrm{e}^{\gamma_{\mathrm{c}} z}+(1-p) \mathrm{e}^{-\gamma_{\mathrm{c}} z} \geqslant 0$.

Consider $f^{\prime \prime}(z)$ :

$f^{\prime \prime}(z)=p \gamma_{\mathrm{c}} \mathrm{e}^{\gamma_{\mathrm{c}} z}-(1-p) \gamma_{\mathrm{pr}} \mathrm{e}^{-\gamma_{\mathrm{pr}} z}$,

which is positive whenever $p>\gamma_{\mathrm{pr}} /\left(\gamma_{\mathrm{c}}+\gamma_{\mathrm{pr}}\right)$. In that case, $f^{\prime}(z)$ is an increasing function, and since $f^{\prime}(0)=1$, we find that $f^{\prime}(z) \geqslant 1$, and therefore $f(z) \geqslant z$ for all $z \geqslant 0$. This proves the first claim. When $p<\gamma_{\mathrm{pr}} /\left(\gamma_{\mathrm{c}}+\gamma_{\mathrm{pr}}\right)$, we will have that $f^{\prime \prime}(z)<0$ for $z \leqslant \widehat{z}$, where $\widehat{z}$ is some positive constant, while $f^{\prime \prime}(z)>0$ elsewhere. Thus, $f^{\prime}(z)$ is decreasing, and next increasing. Since it goes to infinity as an exponential, we need to have that there exists $z_{0}>0$ for which $f\left(z_{0}\right)=z_{0}$. The second claim follows.

Let $z=\bar{\beta}\left(t, T_{1}, T_{2}\right)$ in the Lemma above, and recall that by the definition of $\bar{\beta}\left(t, T_{1}, T_{2}\right)$ it is increasing in $t \leqslant T_{1}$ and decreasing in $T_{1}<t \leqslant T_{2}$. Its maximum is in $t=T_{1}$, where it takes the value $\bar{\beta}\left(T_{1}, T_{1}, T_{2}\right)=\left(1-\mathrm{e}^{-\beta\left(T_{2}-T_{1}\right)}\right) / \beta$. If this maximum is less than $z_{0}$, the jump risk $\theta_{L}\left(t, T_{1}, T_{2}\right)$ will 
be negative for all $t \leqslant T_{2}$. Consider the situation where the maximum is greater than $z_{0}$. Observe that $\bar{\beta}\left(0, T_{1}, T_{2}\right)=$ $\left(\mathrm{e}^{-\beta T_{1}}-\mathrm{e}^{-\beta T_{2}}\right) / \beta$ and $\bar{\beta}\left(T_{2}, T_{1}, T_{2}\right)=0$. If $\bar{\beta}\left(0, T_{1}, T_{2}\right) \geqslant$ $z_{0}$, there exists one $t_{0}$ such that $\bar{\beta}\left(t_{0}, T_{1}, T_{2}\right)=z_{0}$. In this case we find that $\theta_{L}\left(t, T_{1}, T_{2}\right)>0$ for $t<t_{0}$, and $\theta_{L}\left(t, T_{1}, T_{2}\right)<0$ for $t>t_{0}$. If $\bar{\beta}\left(0, T_{1}, T_{2}\right)<z_{0}$, we have the existence of $t_{0}<t_{1}$ being such that $\bar{\beta}\left(t, T_{1}, T_{2}\right)=z_{0}$, $t=t_{0}, t_{1}$. Then $\theta_{L}\left(t, T_{1}, T_{2}\right)$ is negative for $t \leqslant t_{0}$, positive on the interval $t \in\left(t_{0}, t_{1}\right)$ and negative again on $t \in\left(t_{1}, T_{2}\right)$.

Therefore, we may have a situation where the market price of jump risk becomes positive giving a positive contribution to the forward price which makes it larger than the forecasted spot, even in the presence of a negative contribution to the forward price coming from $\theta_{B}$. It is also interesting to see that on the forward curve we can have different signs of the market risk premium depending on how far away from maturity we are on the curve. This change of sign in the market risk premium may only take place when jumps are present in the model, and when the market power of the producer is weaker than his relative risk aversion to the sum of both risk aversion coefficients, see (4.5). Note that when jumps are not present in the spot price, and we have assumed that the market price of risk is constant, the market risk premium will only be either positive or negative, depending on the size of the market power. In general, if we assume that there is only one factor driving the dynamics of the spot price, then the market price of risk ought to change signs in order to get a change in the sign of the market risk premium. Furthermore, if we assume that the market price of risk (per factor) is constant, then we need at least two factors to observe a change in the sign of the market risk premium.

\subsection{Fixed-delivery forwards without spike risk}

To gain further insight into the forward curves implied by the certainty equivalence principle and market power, we consider a spot market without spikes and with a constant level to which the prices mean-revert. Thus, we consider the spot price model

$\mathrm{d} S(t)=(\mu-\alpha S(t)) \mathrm{d} t+\sigma \mathrm{d} B(t)$,

where $\mu$ is a constant, $\alpha>0, \sigma \geqslant 0$, which yields the explicit solution

$S(T)=S(t) \mathrm{e}^{-\alpha(T-t)}+\frac{\mu}{\alpha}\left(1-\mathrm{e}^{-\alpha(T-t)}\right)+\sigma \int_{t}^{T} \mathrm{e}^{-\alpha(T-s)} \mathrm{d} B(s)$

The indifference price of the producer and consumer are defined as

$$
\begin{aligned}
& F_{\mathrm{pr}}^{\gamma_{\mathrm{p}}}(t, T)=-\frac{1}{\gamma_{\mathrm{p}}} \mathbb{E}^{P}\left[\exp \left(-\gamma_{\mathrm{p}} S(T)\right) \mid \mathscr{F}_{t}\right] \quad \text { and } \\
& F_{\mathrm{c}}^{\gamma_{\mathrm{c}}}(t, T)=\frac{1}{\gamma_{\mathrm{c}}} \mathbb{E}^{P}\left[\exp \left(\gamma_{\mathrm{c}} S(T)\right) \mid \mathscr{F}_{t}\right] .
\end{aligned}
$$

A straightforward calculation gives

$$
\begin{aligned}
F_{\mathrm{pr}}^{\gamma_{\mathrm{p}}}(t, T)= & S(t) \mathrm{e}^{-\alpha(T-t)}+\frac{\mu}{\alpha}\left(1-\mathrm{e}^{-\alpha(T-t)}\right) \\
& -\gamma_{\mathrm{p}} \frac{\sigma^{2}}{4 \alpha}\left(1-\mathrm{e}^{-2 \alpha(T-t)}\right)
\end{aligned}
$$

and

$$
\begin{aligned}
F_{\mathrm{c}}^{\gamma_{\mathrm{c}}}(t, T)= & S(t) \mathrm{e}^{-\alpha(T-t)}+\frac{\mu}{\alpha}\left(1-\mathrm{e}^{-\alpha(T-t)}\right) \\
& +\gamma_{\mathrm{c}} \frac{\sigma^{2}}{4 \alpha}\left(1-\mathrm{e}^{-2 \alpha(T-t)}\right) .
\end{aligned}
$$

For fixed delivery contracts the analogous expression to (3.1) becomes

$$
F^{p}(t, T)=p(t, T) F_{\mathrm{c}}^{\gamma_{\mathrm{c}}}(t, T)+(1-p(t, T)) F_{\mathrm{pr}}^{\gamma_{\mathrm{p}}}(t, T)
$$

and a simple calculation implies that

$$
\begin{aligned}
F^{p}(t, T)= & S(t) \mathrm{e}^{-\alpha(T-t)}+\frac{\mu}{\alpha}\left(1-\mathrm{e}^{-\alpha(T-t)}\right) \\
& -p(t, T) \gamma_{\mathrm{p}} \frac{\sigma^{2}}{4 \alpha}\left(1-\mathrm{e}^{-2 \alpha(T-t)}\right) \\
& +(1-p(t, T)) \gamma_{\mathrm{c}} \frac{\sigma^{2}}{4 \alpha}\left(1-\mathrm{e}^{-2 \alpha(T-t)}\right),
\end{aligned}
$$

and the market risk premium becomes

$\pi(t, T)=\left(\gamma_{\mathrm{c}}-p(t, T)\left(\gamma_{\mathrm{p}}+\gamma_{\mathrm{c}}\right)\right) \frac{\sigma^{2}}{4 \alpha}\left(1-\mathrm{e}^{-2 \alpha(T-t)}\right)$.

It is straightforward to see that the sign of $\pi(t, T)$ is given by the sign of $\left(\gamma_{\mathrm{c}}-p(t, T)\left(\gamma_{\mathrm{p}}+\gamma_{\mathrm{c}}\right)\right)$. Therefore, when $p(t, T)<\gamma_{\mathrm{c}} /\left(\gamma_{\mathrm{p}}+\gamma_{\mathrm{c}}\right)$ we have that $\pi(t, T)>0$ and vice versa.

Note that we may also write the expression of the forward as:

$$
\begin{aligned}
F^{p}(t, T)= & \frac{\mu}{\alpha}+\frac{\sigma^{2}}{4 \alpha}\left(p(t, T)\left(\gamma_{\mathrm{p}}+\gamma_{\mathrm{c}}\right)-\gamma_{\mathrm{p}}\right) \\
& +\left(S(t)-\frac{\mu}{\alpha}\right) \mathrm{e}^{-\alpha(T-t)}\left(p(t, T)\left(\gamma_{\mathrm{p}}+\gamma_{\mathrm{c}}\right)-\gamma_{\mathrm{p}}\right) \mathrm{e}^{-2 \alpha(T-t)},
\end{aligned}
$$

and observe that the forward price consists of three terms, a "constant" level, a "slow" mean-reversion level $\exp (-\alpha(T-t))$ and a "fast" mean-reversion level $\exp (-2 \alpha(T-t))$. Moreover, if we assume a constant market power $p$ with

$p<\frac{\gamma_{\mathrm{pr}}}{\gamma_{\mathrm{pr}}+\gamma_{\mathrm{c}}}$

then the last term in (4.10) will be exponentially increasing towards zero. Further, if $S(t)>\mu / \alpha$, then the $\exp (-\alpha(T-t))$-term will be "slowly" decreasing to zero. In effect, we produce a hump in the forward curve. This hump will be in the short end of the curve.

\section{Empirical evidence: The German market}

In this section we apply our model to the German electricity market. We do this in two steps. First we estimate the physical parameters of a two-factor model. Second, 
using forward market data, denoted by $F\left(t, T_{1}, T_{2}\right)$, we estimate the risk-aversion coefficients for both producers and consumers and estimate the producer's market power.

We employ daily spot prices, the so-called Phelix base load traded at the EEX, and prices for forward contracts with different delivery periods: monthly, quarterly and yearly. Our data covers the period January 22002 to January 12006 where we have 1461 spot price observations. The forward data consist of 108 contracts with monthly delivery, 35 contracts with quarterly delivery and 12 contracts with yearly delivery.

We apply the model to

$S(t)=\Lambda(t)+X(t)+Y(t)$

where, as described above, $\Lambda(t)$ is the seasonal component,

$$
\begin{array}{r}
\mathrm{d} X(t)=-\alpha X(t) \mathrm{d} t+\sigma \mathrm{d} B(t) \\
\mathrm{d} Y(t)=-\beta Y(t) \mathrm{d} t+\mathrm{d} L(t)
\end{array}
$$

where $\alpha \geqslant 0, \beta \geqslant 0 \sigma \geqslant 0, B(t)$ is a standard Brownian motion and

$L(t)=\sum_{i}^{N(t)} J_{i}$

is a compound Poisson process. $N(t)$ is a homogeneous Poisson process with intensity $\lambda$ and $J_{i}$ 's are i.i.d. with exponential density function

$f(j)=p \lambda_{1} \mathrm{e}^{-\lambda_{1} j} \mathbf{1}_{j>0}+(1-p) \lambda_{2} \mathrm{e}^{-\lambda_{2}|j|} \mathbf{1}_{j<0}$,

where $\lambda_{1}>0$ and $\lambda_{2}>0$ are responsible for the decay of the tails for the distribution of positive and negative jump sizes and $\mathbf{1}$ is the indicator function. Finally we assume that $N(t), J$ and $B(t)$ are independent.

We remark in passing that a two-factor model as we consider here will in general violate the no-arbitrage condition for the forward market if this consists of more than two contracts (recall the discussion in Section 4). However, the purpose of the empirical study is to provide an insight into the market power and the market risk premium, and thus we choose a simple and tractable model to analyze. We may, on the other hand, to be consistent with the noarbitrage conditions mentioned above, split the Brownian motion part of $X(t)$ into several factors to ensure an arbitrage-free market as required by our conditions above. Therefore, this means that we must choose the same number of Brownian motions as number of contracts and our results to follow will not change with this modification.

To be able to estimate the seasonal component and the parameters of the OU and jump processes we follow a procedure similar to that in Cartea and Figueroa (2005) and Lucía and Schwartz (2002). Therefore, for the seasonal component we assume

$$
\begin{aligned}
\Lambda(t)= & a_{0}+a_{1} \mathbf{1}_{\{t=S u\}}+a_{2} \mathbf{1}_{\{t=M o\}}+a_{3} \mathbf{1}_{\{t=T u, W e, T h\}}+a_{4} \mathbf{1}_{\{t=S a\}} \\
& +a_{5} \cos \left[\frac{6 \pi}{365}\left(t+a_{6}\right)\right]+a_{7} t,
\end{aligned}
$$

Table 1

Estimated coefficients of $\Lambda(t)$

\begin{tabular}{lllllllll}
\hline$a_{0}$ & $a_{1}$ & $a_{2}$ & $a_{3}$ & $a_{4}$ & $a_{5}$ & $a_{6}$ & $a_{7}$ & $\begin{array}{l}\text { Squared } \\
\text { error }\end{array}$ \\
\hline 19.43 & -11.43 & 0.78 & 2.13 & -6.13 & 1.2 & 53.64 & 0.016 & 39420.74 \\
\hline
\end{tabular}

Table 2

Parameter estimates for OU and jump components

\begin{tabular}{lllllll}
\hline$\alpha$ & $\sigma$ & $p$ & $\lambda$ & $\lambda_{1}$ & $\lambda_{2}$ & $\beta$ \\
\hline 0.44 & 5.2 & 0.72 & 0.054 & 0.031 & 0.053 & 0.2 \\
\hline
\end{tabular}

Table 3

Empirical ad simulated moments (1000 paths)

\begin{tabular}{lllll}
\hline & Mean & Standard deviation & Skewness & Kurtosis \\
\hline Empirical $S(t)$ & 31.6 & 15.2 & 2.7 & 14.5 \\
Simulated $S(t)$ & 32.1 & 15.6 & 2.2 & 13.8 \\
\hline
\end{tabular}

where the indicator function is acting on the different days of the week. The parameter estimates for the seasonal component are shown in Table 1 and the estimates for the OU and Jump components are shown in Table 2. ${ }^{5}$ Moreover, Table 3 shows the mean, standard deviation, skewness and kurtosis of the spot prices and those resulting from 10,000 paths using our model and Fig. 5 shows the realized and a simulated path. It is clear that our model captures both statistical and trajectile properties.

In order to calculate the market power $p\left(t, T_{1}, T_{2}\right)$ and the forward premium $\pi\left(t, T_{1}, T_{2}\right)$ we need to choose the risk aversion coefficients for the consumer and the producer, $\gamma_{\mathrm{c}}$ and $\gamma_{\mathrm{pr}}$, respectively. Since $F_{\mathrm{c}}\left(t, T_{1}, T_{2}\right)$ (upper bound) and $F_{\mathrm{pr}}\left(t, T_{1}, T_{2}\right)$ (lower bound) depend on the choice of $\gamma_{\mathrm{c}}$ and $\gamma_{\mathrm{pr}}$, we estimate $\gamma_{\mathrm{pr}}$ and $\gamma_{\mathrm{c}}$ by minimizing the distance between $F_{\mathrm{c}}\left(t, T_{1}, T_{2}\right), F_{\mathrm{pr}}\left(t, T_{1}, T_{2}\right)$ and the market prices of forwards $F\left(t, T_{1}, T_{2}\right)$, respectively, in the following way.

We examine the time range $t=1=02 / \mathrm{Jan} / 2002$ until $t=1461=31 / \mathrm{Dec} / 2005$. For most days (excluding weekends and holidays) we have prices for forward contracts with delivery one month, three months (quarter) and twelve months (year). As long as there is a price on day $t$, we determine all values of $\gamma_{\mathrm{pr}}$ and $\gamma_{\mathrm{c}}$ such that

$F_{\mathrm{pr}}\left(t, T_{1}, T_{2}\right) \leqslant F\left(t, T_{1}, T_{2}\right) \leqslant F_{\mathrm{pr}}\left(t, T_{1}, T_{2}\right)$

and introduce intervals which contain the risk aversion parameters. For all trading days $t \in[1,1461]$, we define the intervals $I_{\mathrm{pr}}^{t}$ and $I_{\mathrm{c}}^{t}$ containing values for $\gamma_{\mathrm{pr}}$ and $\gamma_{\mathrm{c}}$ by guaranteeing that (5.2) holds. Thus, to find the ranges for the parameters of risk aversion we implement the following algorithm:

- $t=1$ : Determine valid intervals $I_{\mathrm{pr}}^{1}$ and $I_{\mathrm{c}}^{1}$ such that $F_{\mathrm{pr}}\left(1, T_{1}, T_{2}\right) \leqslant F\left(1, T_{1}, T_{2}\right) \leqslant F_{\mathrm{c}}\left(1, T_{1}, T_{2}\right)$, for all delivery periods $\left[T_{1}, T_{2}\right]$, traded on day 1 .

\footnotetext{
${ }^{5}$ For more details on the estimation procedure see (Metka, 2007).
} 

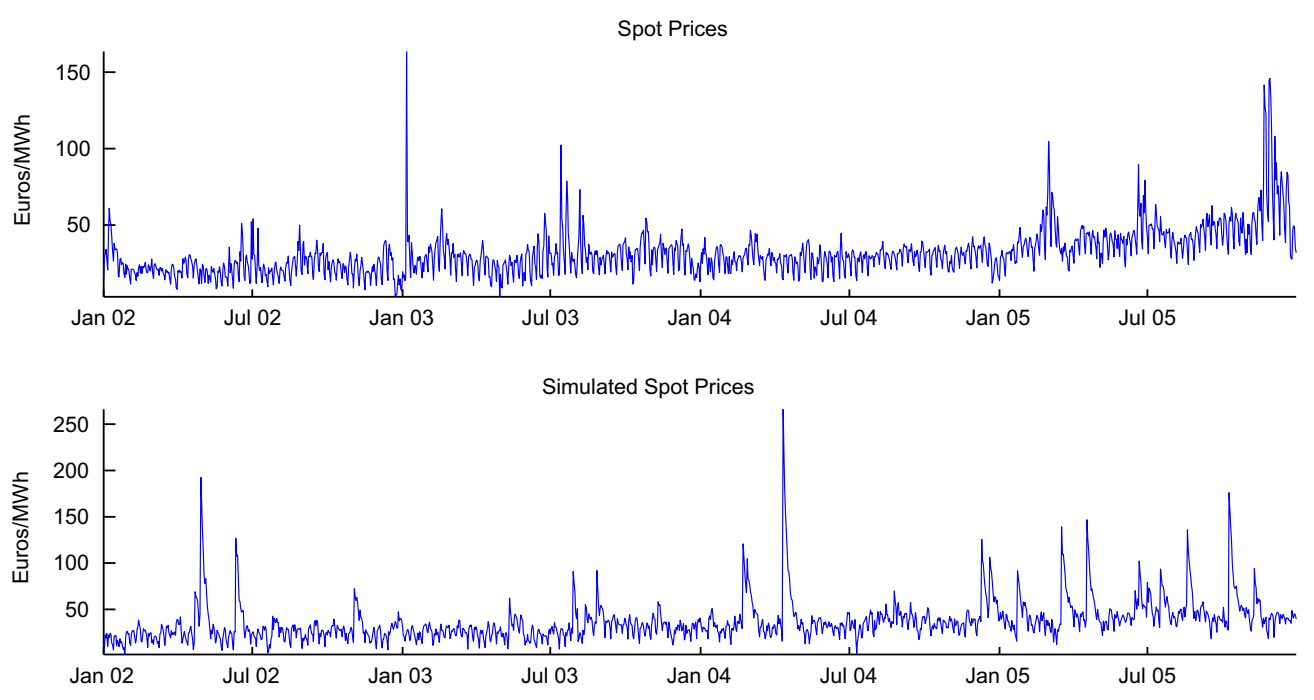

Fig. 5. Spot and simulated spot prices.

- $t=2$ : Determine valid intervals $I_{\mathrm{pr}}^{2}$ and $I_{\mathrm{c}}^{2}$ such that $F_{\mathrm{pr}}\left(2, T_{1}, T_{2}\right) \leqslant F\left(2, T_{1}, T_{2}\right) \leqslant F_{\mathrm{c}}\left(2, T_{1}, T_{2}\right)$, for all delivery periods $\left[T_{1}, T_{2}\right]$, traded on day 2 .

-...

- $t=1461$ : Determine valid intervals $I_{\mathrm{pr}}^{1461}$ and $I_{\mathrm{c}}^{1461}$ such that $F_{\mathrm{pr}}\left(1461, T_{1}, T_{2}\right) \leqslant F\left(1461, T_{1}, T_{2}\right) \leqslant F_{\mathrm{c}}\left(1461, T_{1}, T_{2}\right)$, for all delivery periods $\left[T_{1}, T_{2}\right]$, traded on day 1461 .

This algorithm guarantees that no forward prices $F\left(t, T_{1}, T_{2}\right)$ will lay outside the bounds $F_{\mathrm{pr}}\left(t, T_{1}, T_{2}\right)$ and $F_{\mathrm{c}}\left(t, T_{1}, T_{2}\right)$. Consequently the results show that $\gamma_{\mathrm{pr}} \in[0.421, \infty)$ and $\gamma_{\mathrm{c}} \in[0.701, \infty)$. In our calculations we choose $\gamma_{\mathrm{pr}}=0.421$ and $\gamma_{\mathrm{c}}=0.701$ which seems a reasonable working assumption where producers are less risk-averse than consumers.

We continue by recalling that the market power and market risk premium are given by

$p\left(t, T_{1}, T_{2}\right)=\frac{F\left(t, T_{1}, T_{2}\right)-F_{\mathrm{pr}}\left(t, T_{1}, T_{2}\right)}{F_{\mathrm{c}}\left(t, T_{1}, T_{2}\right)-F_{\mathrm{pr}}\left(t, T_{1}, T_{2}\right)}$

and

$\pi\left(t, T_{1}, T_{2}\right)=F\left(t, T_{1}, T_{2}\right)-\mathbb{E}^{P}\left[\frac{1}{T_{2}-T_{1}} \int_{T_{1}}^{T_{2}} S(u) \mathrm{d} u \mid \mathscr{F}_{t}\right]$.
To calculate $p\left(t, T_{1}, T_{2}\right)$ and $\pi\left(t, T_{1}, T_{2}\right)$ we split the data in three non-overlapping periods. Table 4 lists the contracts we employ in every period. The key criterion is to include in the first period all contracts that were being traded on January 2 2002. Then the second period starts when all contracts that were being traded on January 22002 are no longer traded. Finally, the third period is defined in a similar way.

Figs. 6-8 show our results when all forward contracts that were being traded on January 22002 are taken into account. In other words we show the results from 18 monthly contracts, 7 quarterly contracts and 3 yearly contracts (see Table 4).

In particular, Fig. 6 shows the results for the 18 monthly contracts that were trading on January 2 2002. As expected we observe a decline in the producer's market power as time to delivery increases. For example, in the contract with delivery period closest to January 22002 the producer's market power is slightly over 0.80 and decreases to values below 0.30 corresponding to contracts that start delivery on or after March 2003. Moreover, Figs. 7 and 8 show the same behavior: a decaying market power for producers, as time to delivery increases, for both quarterly and yearly forward contracts.

Table 4

Forward contracts

\begin{tabular}{|c|c|c|c|c|}
\hline$t$ & Type & \# Contracts & Delivery periods & $F\left(t, T_{1}, T_{2}\right)$ \\
\hline 01/Jan/2002 & Monthly & 18 & Jan 2002-May 2003 & $F\left(2, T_{1}, T_{2}\right)$ \\
\hline 01/Jan/2002 & Quarterly & 7 & 2nd qtr 2002-4th qtr 2003 & $F\left(2, T_{1}, T_{2}\right)$ \\
\hline 01/Jan/2002 & Yearly & 3 & 2003-2005 & $F\left(2, T_{1}, T_{2}\right)$ \\
\hline 03/Mar/2003 & Quarterly & 7 & 2nd qtr 2003-4th qtr 2004 & $F\left(400, T_{1}, T_{2}\right)$ \\
\hline 03/Mar/2003 & Yearly & 3 & 2004-2006 & $F\left(400, T_{1}, T_{2}\right)$ \\
\hline 04/Oct/2005 & Monthly & 7 & Oct 2005-Apr 2006 & $F\left(1373, T_{1}, T_{2}\right)$ \\
\hline
\end{tabular}



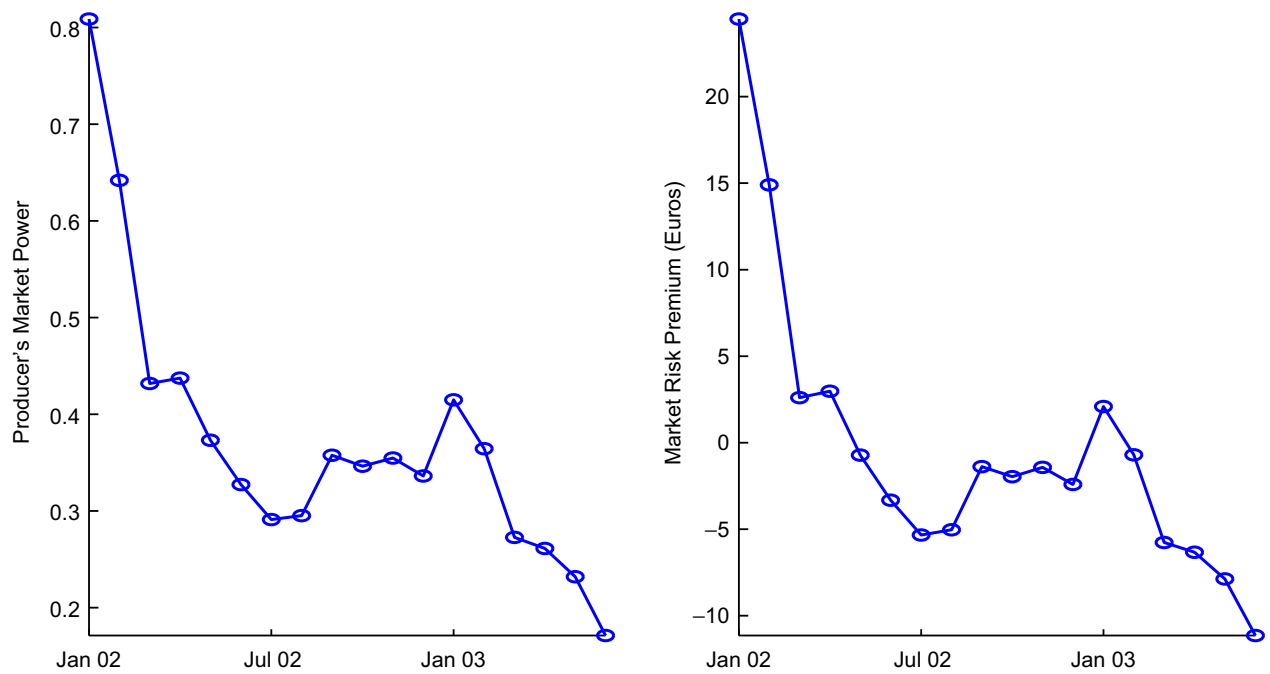

Fig. 6. Producer's market power and market risk premium, 18 monthly contracts with $t=$ January 22002 .
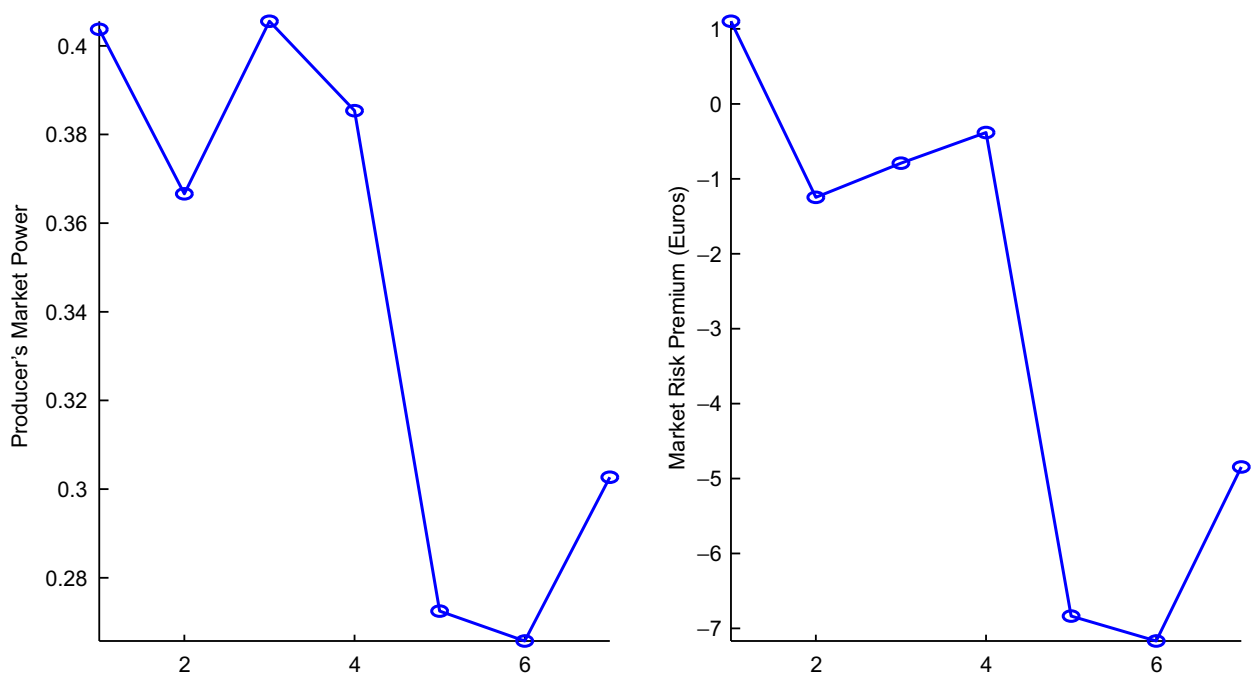

Fig. 7. Producer's market power and market risk premium, 7 quarterly contracts with $t=$ second quarter 2002.
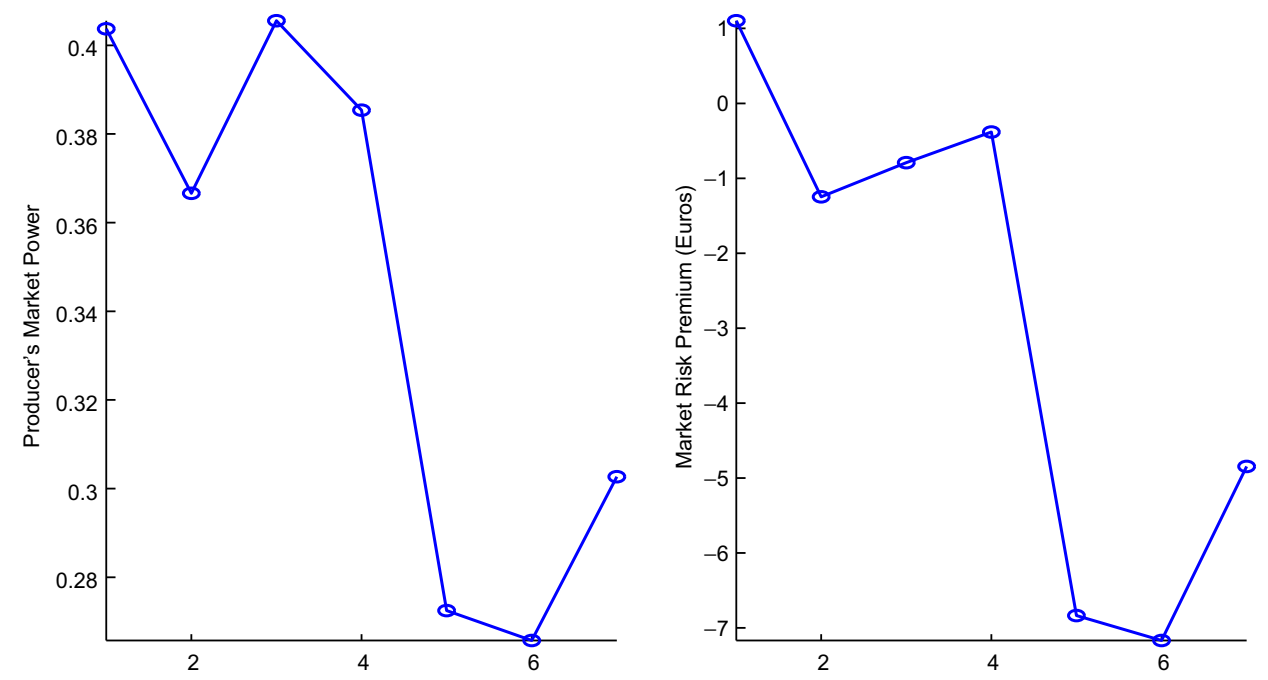

Fig. 8. Producer's market power and market risk premium, 3 yearly contracts with $t=2002$. 
Moreover, as predicted by our theoretical examples above, the producer's market power is much higher in the short end for monthly contracts, Fig. 6, than for quarterly and yearly, Figs. 7 and 8 . In the short-term, the presence of jumps incentivies consumer's to hedge against risk produced by upward spikes, i.e. exert upward pressure on demand for forwards, whilst at the same time, producer's have less incentives to sell forwards when positive spikes are present. Hence, we see that monthly forward contracts, that start delivery relatively soon, trade at a high market risk premium which in our framework is also reflected in a high market power for producers. Furthermore, the further away the start of the delivery period is, the presence of price spikes, due to the fast mean reverting nature of the spot price, poses negligible risks. This situation is evident in the term structure of the producer's market power and market risk premium obtained from the quarterly and yearly contracts which are roughly the same in both cases (see Figs. 7 and 8).

We also depict results for the market risk premium. We see that the risk premium $\pi\left(t, T_{1}, T_{2}\right)$ shows a clear term structure. As expected, $\pi\left(t, T_{1}, T_{2}\right)$ is decreasing in $\left[T_{1}, T_{2}\right]$, i.e. the further the delivery of the contract is, the smaller the risk premium. In our model, this result can be explained by the decreasing market power that producers have; the larger the difference $T_{1}-t$, the keener producers will be, relative to consumers, to trade forwards. Hence, forward prices will move away from the upper bound $F_{\mathrm{c}}\left(t, T_{1}, T_{2}\right)$. As a result, the risk premium $\pi\left(t, T_{1}, T_{2}\right)$ is decreasing, and at some point in time, it becomes negative. Finally, it is interesting to compare the point in time where the risk premium changes its sign. When looking at the term structure of $\pi\left(t, T_{1}, T_{2}\right)$ obtained from monthly and quarterly contracts we see that the change of sign occurs around the months of April and May.

\section{Conclusions}

In this article we address the important question of what gives rise to the market risk premium. We provide a framework that allows us to explain how risk preferences of market players explain the sign and magnitude of the market risk premium across different forward contract maturities. A crucial step in our framework is to be able to incorporate the relative eagerness consumers and producers show, via their respective representative agents, to enter forward contracts. We show that there is an attainable set where consumers and producers are willing to strike a deal, but it is this eagerness to hedge risk across different points in time, which we label market power, what singles out a unique equilibrium price for each forward contract.

Furthermore, these equilibrium prices that belong to the attainable set must clearly correspond to those obtained from pricing forwards under a risk-neutral measure. Therefore, as an illustration of our approach, we looked at wholesale electricity prices and were able to make an expli- cit analytical connection between the market prices of risk and market power with the degrees of risk aversion of the representative agents. To exemplify our methodology further, we looked at particular examples where it was straightforward to see how different sources of risk, for instance jump or diffusion risk, contribute to the market risk premium and we were able to obtain and explain the very distinctive characteristics observed in electricity markets as well as in other markets such as gas, oil, etc.

We apply our model to the German electricity market. Our empirical results endorse our theoretical predictions. For instance, we find that over short-term horizons, and in the presence of spike risk, producer's market power is at its highest. For example, in the contracts with delivery period closest to January 22002 the producer's market power is around 0.80 and decreases to values below 0.30 corresponding to contracts that start delivery on or after March 2003. This situation is also reflected in the market risk premium. Monthly contracts that mature in the near future, trade at a high premium, figures in excess of 15 Euros. And monthly contracts that start delivery in a relatively long period of time, for instance in 6 months or longer, trade most of the time, at a high discount.

Finally, we generally observe that for each class of contract (monthly, quarterly or yearly) the producer's market power and the market risk premium show a term structure that is decreasing as time to maturity of the forward contract increases.

\section{References}

Benth, Fred Espen, Kallsen, Jan, Meyer-Brandis, Thilo, 2007. A nonGaussian Ornstein-Uhlenbeck process for electricity spot price modelling and derivatives pricing. Applied Mathematical Finance 14 (2), 153-169.

Cartea, Álvaro, Figueroa, Marcelo G., 2005. Pricing in electricity markets: A mean reverting jump diffusion model with seasonality. Applied Mathematical Finance 12 (4), 313-335.

Cartea Álvaro, Williams Thomas, 2007. UK gas markets: The market price of risk and applications to multiple interruptible supply contracts. Energy Economics, in press.

Cont, R., Tankov, P., 2004. Financial modelling with jump processes, first ed.. In: Financial Mathematics Series Chapman and Hall, London.

Lucía, J., Schwartz, E., 2002. Electricity prices and power derivatives: Evidence from the nordic power exchange. Review of Derivatives Research 5, 5-50.

Merton, R., 1990. Continuous - Time Finance, first ed. Blackwell.

Metka, K., 2007. Pricing Forward Contracts in Power Markets by the Certainty Equivalent Principle: Explaining the Sign of the Market Risk Premium. Diplomarbeit, University of ULM.

Protter, Philip, 1992. Stochastic integration and differential equations, a new approach, second ed.. In: Applications of Mathematics, vol. 21 Springer-Verlag.

Sato, K.I., 1999. Lévy processes and infinitely divisible distributions, first ed.. In: Cambridge Studies in advanced mathematics, vol. 68 Cambridge University Press, Cambridge.

Schwartz, E., Smith, J., 2000. Short-term variations and long-term dynamics in commodity prices. Management Science 46 (7), 893-911.

Schwartz, Eduardo, 1997. The stochastic behavior of commodity prices: Implications for valuation and hedging. Journal of Finance 52 (3), 923-973. 